\title{
A Novel Interplanetary Communications Relay
}

\author{
Robert J. McKay ${ }^{1}$, Malcolm Macdonald ${ }^{2}$ \\ Advanced Space Concepts Laboratory, University of Strathclyde, Glasgow G1 1XJ, United Kingdom
}

Massimiliano Vasile ${ }^{3}$

Space Advanced Research Team, University of Glasgow, Glasgow G21 8QQ, United Kingdom

Francois Bosquillon de Frescheville ${ }^{4}$

European Space Operations Centre, European Space Agency, 64293 Darmstadt, Germany

\begin{abstract}
A case study of a potential Earth-Mars interplanetary communications relay, designed to ensure continuous communications, is detailed. The relay makes use of orbits based on artificial equilibrium points via the application of continuous low thrust, which allows a spacecraft to hover above the orbital plane of Mars and thus ensure communications when the planet is occulted with respect to the Earth. The artificial equilibria of two different lowthrust propulsion technologies are considered: solar electric propulsion, and a solar sail/solar electric propulsion hybrid. In the latter case it is shown that the combination of sail and solar electric propulsion may prove advantageous, but only under specific circumstances of the relay architecture suggested. The study takes into account factors such as the spacecraft's power requirements and communications band utilized to determine the mission and system architecture. A detailed contingency analysis is considered for recovering the relay after increasing periods of spacecraft motor failure, and combined with a consideration for how best to deploy the relay spacecraft to maximise propellant reserves and mission duration.
\end{abstract}

\section{Nomenclature}

$A_{S} \quad$ sail area (not including thin film solar array area)

$A_{T F} \quad$ thin film area

$A_{T} \quad$ total hybrid sail area, $=A_{S}+A_{T F}$

$a_{r e f}$ dimensional reference acceleration, equal to unit sail lightness number $=5.93 \mathrm{mms}^{-2}$

$\boldsymbol{a}_{g c}$ nondimensional required acceleration vector to balance gravitational and centrifugal force

$a_{s} \quad$ nondimensional acceleration magnitude due to solar radiation pressure

$\boldsymbol{a}_{s} \quad$ nondimensional acceleration due to solar radiation pressure

$\boldsymbol{a}_{S E P}$ nondimensional acceleration due to SEP thruster

\footnotetext{
${ }^{1}$ Research Fellow, Advanced Space Concepts Laboratory, University of Strathclyde, Glasgow, UK.

2 Associate Director, Advanced Space Concepts Laboratory, University of Strathclyde, Glasgow, UK, AIAA Associate Fellow.

${ }^{3}$ Senior Lecturer, Space Advanced Research Team, University of Glasgow, Glasgow, UK.

4 Future Studies Operations Concept Engineer, European Space Operations Centre, Human Spaceflight and Explortation Department, Darmstadt, Germany
} 
$a_{T} \quad$ nondimensional thrust acceleration from electric propulsion system of hybrid sail

$\boldsymbol{a}_{g c} \quad$ nondimensional acceleration required to balance gravitational and centrifugal

$I_{S P} \quad$ specific impulse

$m \quad$ spacecraft mass

$m_{0} \quad$ initial mass of hybrid sail

n thrust vector orientation

$\boldsymbol{r}, r$ position vector with respect to centre of mass of primaries, orbit radius

$\tilde{r}_{S} \quad$ solar sail film reflectivity

$\tilde{r}_{T F} \quad$ thin film solar array reflectivity

$T \quad$ continuous and constant low thrust

$v \quad$ centripetal potential

$\boldsymbol{V} \quad$ augmented potential

$\alpha \quad$ pitch angle

$\beta_{0} \quad$ solar sail lightness number

$\lambda \quad$ ratio of $\|\boldsymbol{a}\|$ to $\|\nabla V\|$

$\delta \quad$ solar sail clock angle

$\tilde{\delta} \quad$ clock angle of vector $\boldsymbol{a}_{g c}$

$\theta \quad$ solar sail cone angle

$\tilde{\theta} \quad$ cone angle of vector $\boldsymbol{a}_{g c}$

$\mu \quad$ reduced mass gravitational parameter

$\boldsymbol{\omega}$ orbital angular velocity

$\sigma^{*} \quad$ critical solar sail loading parameter, $=1.53 \times 10^{-3} \mathrm{~kg} \mathrm{~m}^{-2}$

\section{1 - Introduction}

The concept of counter-acting gravity through a thrust vector was apparently first proposed by Dusek in 1966, who noted that a spacecraft could be held in an artificial equilibrium at a location some distance from a natural libration point if the difference in gravitation and centripetal force (gravity gradient) were compensated for by continuous low thrust propulsion [1]. More recently, this concept has been explored for the special case of solar sail 
propelled spacecraft which can, in principle, generate continuous thrust without the need for reaction mass [2]. A generic continuous thrust can be applied in all directions including perpendicular to the flight direction, which forces the spacecraft out of a natural orbit into a displaced, non-Keplerian orbit: such orbits could have a diverse range of potential applications. Forward coined the term "statite" [3] in reference to a mission using a solar sail to hover above, or below, the Earth in such a displaced orbit in a concept which has evolved to become known as the Polar Observer, or PoleSitter, mission [4]. Following the work of Forward, $\mathrm{M}^{\mathrm{c}}$ Innes made an extensive study of the concept [4], exploring new regions of interest, including the study of artificial, or displaced, Lagrange points which was considered extensively in the late 1990's under the NASA/JPL/NOAA GeoStorm mission concept [5,6].

The initial work led by $\mathrm{M}^{\mathrm{c}}$ Innes has since been evolved by others to consider different forms of propulsion, such as solar electric propulsion (SEP) [7,8]. Such work has focused primarily on Earth-centred trajectories, although some authors have considered individual applications of non-Keplerian orbits outwith the Earth's influence - for example, for in-situ observation of Saturn's rings [9]. This prompted McKay et al. to extend the work of McInnes for celestial bodies other than just the Earth, and systematically catalogue non-Keplerian orbit regions throughout the solar system [10].

In doing so, Ref. [10] highlighted the possibility of using such orbits for a potential Earth-Mars communications relay, not dissimilar in concept to that proposed for lunar communications by Wawrzyniak \& Howell [11]. For any future manned exploration of Mars, continuous communication between the surfaces of the two planets will be required. Currently, during periods of solar occultation assets both in-orbits about Mars and on its surface are out of communication with ground controllers. While such a scenario is undesirable for robotic assets it is acceptable. However, this is not so for human exploration. Therefore, a communication relay is required to ensure continuous communication between Earth and Mars. To address this issue non-Keplerian orbits outwith the orbital plane of Mars were considered in Ref. [10], and expanded upon here. Indeed, it is noted that any spacecraft within, or even which passes through, the orbital plane of a planet in the solar system shall experience periods of solar occultation of Earth, and thus the problem is more generic than the specific case of Mars.

\section{2 - Highly Non-Keplerian orbit model and definition}

Following McInnes [12], the conditions for circular displaced highly non-Keplerian orbits can be investigated by considering the dynamics of a spacecraft of mass $m$ in a reference frame $\boldsymbol{R}(x, y, z)$ rotating at constant angular velocity $\boldsymbol{\omega}$ relative to an inertial frame $\boldsymbol{I}(X, Y, Z)$. With such a system the equations of motion of the spacecraft are given by,

$$
\ddot{\boldsymbol{r}}+2 \boldsymbol{\omega} \times \dot{\boldsymbol{r}}+\nabla \boldsymbol{V}=\boldsymbol{a}
$$


where $\boldsymbol{r}$ is the position vector of the spacecraft, dots denote differentiation with respect to time $t$, and $\boldsymbol{V}$ and $\boldsymbol{a}$ are the augmented potential and the continuous and constant acceleration due to the propulsion system respectively, the former being given by,

$$
\boldsymbol{V}=-\left[\left(\frac{1-\mu}{\left\|\boldsymbol{r}_{1}\right\|}+\frac{\mu}{\left\|\boldsymbol{r}_{2}\right\|}\right)\right]+\frac{1}{2}\|\boldsymbol{\omega} \times \boldsymbol{r}\|^{2}
$$

in units where the gravitational constant $G=1$ and the system has total unit mass, and where $\mu$ is the reduced mass,

$$
\mu=\frac{m_{1}}{m_{1}+m_{2}}
$$

and the latter being given by,

$$
\boldsymbol{a}=\left[\frac{T}{m}\right] \boldsymbol{n}
$$

where $\boldsymbol{n}$ is the direction of the thrust $T$ (where this equation makes no assumption about the propulsion system used, so for e.g. a solar sail Equation (4) must be modified accordingly). By setting $\ddot{\boldsymbol{r}}=\dot{\boldsymbol{r}}=0$, i.e. assuming equilibrium conditions in the rotating frame, then the equation $\nabla \boldsymbol{V}=\boldsymbol{a}$ defines a surface of equilibrium points. Further, the required thrust vector orientation for an equilibrium solution is then given by,

$$
\boldsymbol{n}=\frac{\nabla \boldsymbol{V}}{\|\nabla \boldsymbol{V}\|}
$$

and the magnitude of the thrust vector, $\|\boldsymbol{a}\|$, is given by,

$$
\|\boldsymbol{a}\|=\|\nabla \boldsymbol{V}\|
$$

Equation (6) provides a simple definition of what constitutes a non-Keplerian orbit. Defining a parameter $\lambda$ such that,

$$
\lambda=\frac{\|\boldsymbol{a}\|}{\|\nabla \boldsymbol{V}\|}
$$


the specific case of highly non-Keplerian orbits can be considered: that is, when $\lambda \cong 1$, or, equivalently, when the acceleration applied by the low-thrust propulsion system is of approximately the same order as the gravitational acceleration experienced by the spacecraft. For comparison, orbits with $\lambda=0$ represent ideal Keplerian orbits, in essence the large subset of classical celestial orbital mechanics without the addition of a non-conservative force.

McKay et al. [13] carried out a detailed study of artificial equilibrium points and surfaces throughout the solar system, formulating the equilibrium points in the circular restricted three-body problem (CRTBP). This was done by considering the dynamics of a spacecraft of mass $m$ in a rotating reference frame in which the primary masses $m_{1}$ and $m_{2}$ are fixed in the rotating frame (as shown in Figure 1), or, equivalently, in the inertial frame they orbit circularly with constant angular velocity $\omega$ in the ecliptic plane about their common centre of mass. If the unit of length of the system is chosen such that the distance $R$ between the primary masses is unity, and with $G=1$ and reduced mass as defined by Equation (3), then the nondimensional unit acceleration of the system is given by $a_{\text {ref }}=\omega^{2} R$. In Figure 1 the $x$-axis points between the primary masses, the $y$-axis denotes the axis of rotation and the $z$-axis is orthogonal to both.

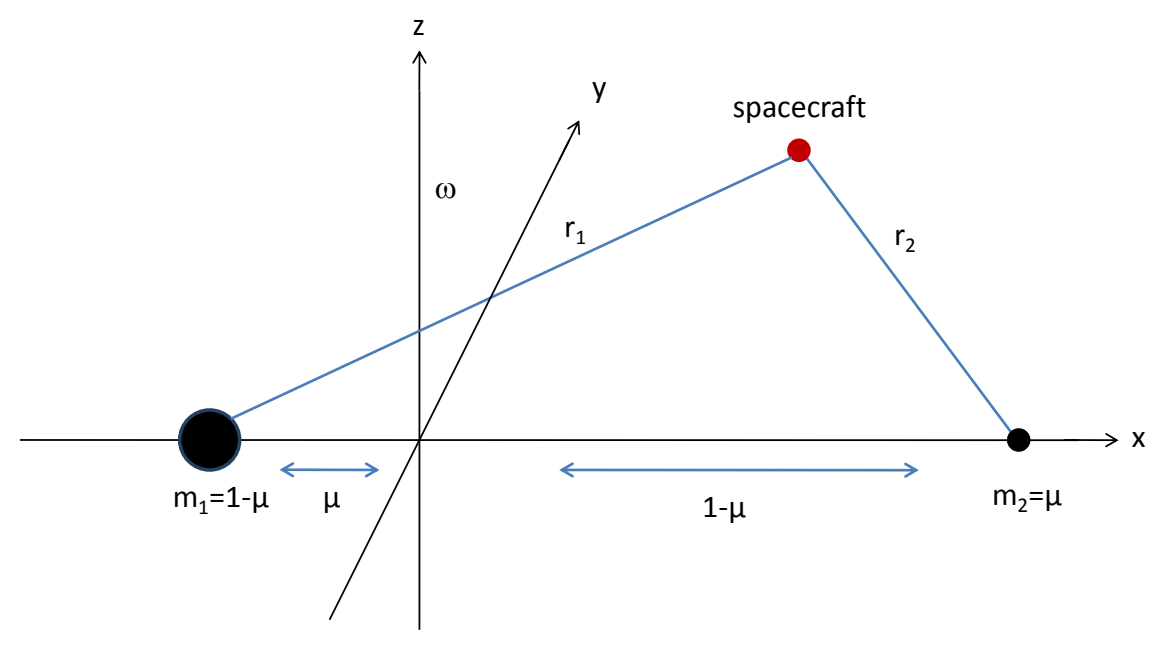

Fig. 1: The rotating coordinate frame and the spacecraft position therein for the restricted three-body problem.

The position vectors $\boldsymbol{r}_{1}$ and $\boldsymbol{r}_{2}$ of the spacecraft with respect to the primary masses $m_{1}$ and $m_{2}$ are then given by $\boldsymbol{r}_{1}=(x+\mu, y, z)^{T}$ and $\boldsymbol{r}_{2}=((x-(1-\mu)), y, z)^{T}$ respectively, where the equations of motion, augmented potential and reduced mass of the system are as previously given by Equations (1-3). The equation for the magnitude of the acceleration vector $\|\boldsymbol{a}\|$ as given by Equation (6) then defines an implicit function in the rotating coordinates. As an implicit function can be expressed in the form $f(x, y, z)=0$ it defines a three-dimensional equithrust surface (or, equivalently, two-dimensional equithrust contours) which can be plotted, as illustrated in Figure 2 for the immediate region around Mercury. 
Anywhere on this equithrust surface, a spacecraft with the required thrust, oriented in the direction needed, will therefore exist in equilibrium with the body in question. With such a formulation then a spacecraft occupying a given artificial Lagrange point on this surface tracks out a circular periodic orbit in the inertial reference frame, with the period being defined by that of the body which the spacecraft is in equilibrium with.
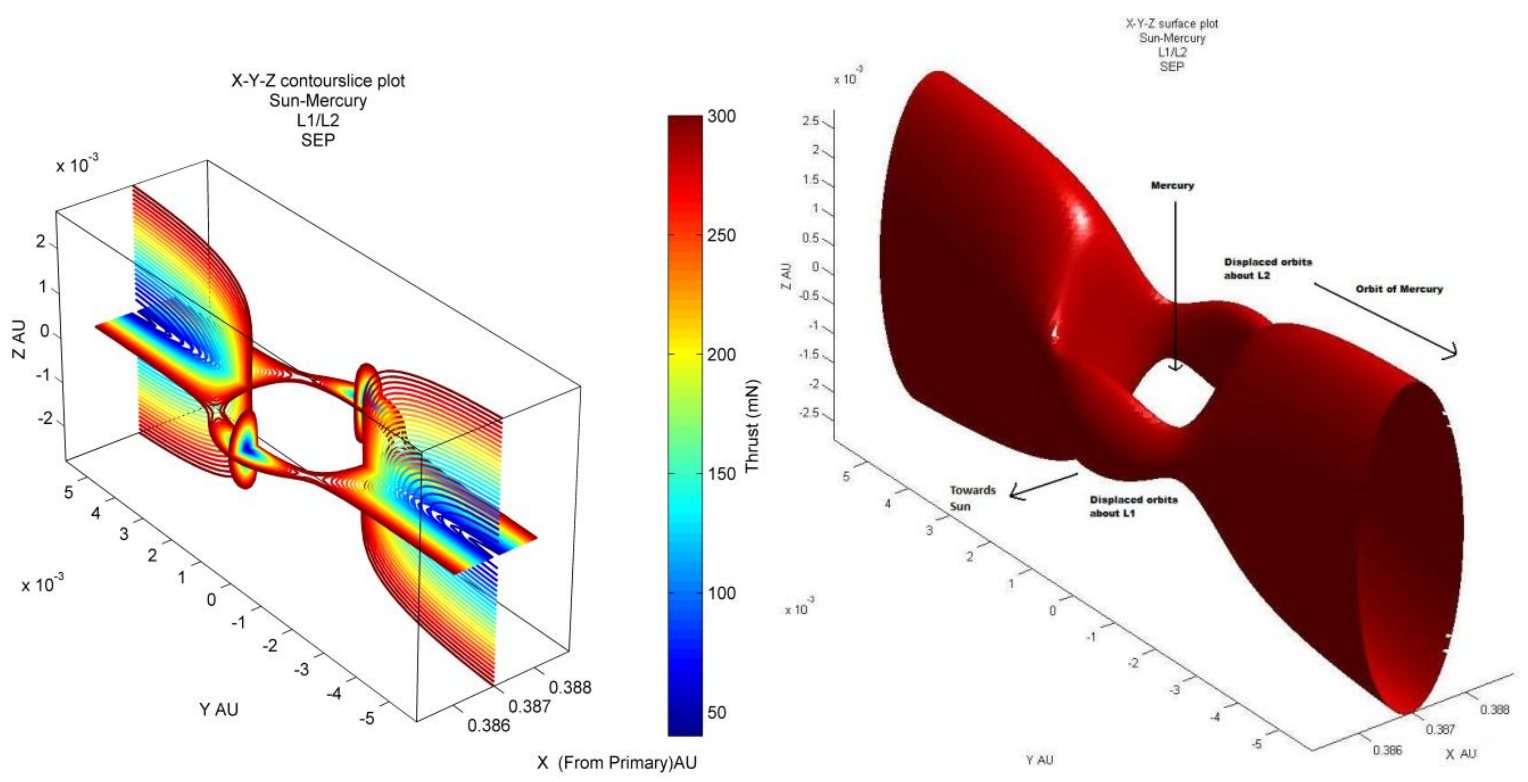

Fig. 2: Artificial equilibrium points depicted by equithrust contours projected onto the planes perpendicular to and parallel to the orbital plane (left), and the $300 \mathrm{mN}$ equithrust surface (right) for the Sun-Mercury $\mathrm{L}_{1} / \mathrm{L}_{2}$ system.

\section{$\underline{3 \text { - Communication Relay Architecture Options }}$}

\subsection{Overview}

As referred to previously, McKay and co-workers [10] outlined a concept for an Earth-Mars communications relay that uses non-Keplerian orbits to hover above the orbital plane of Mars in order to relay signals between the two bodies during periods of occultation by the Sun, and thus ensure continuous communications between Earth and assets on the surface of Mars. Here that work is recapped briefly, prior to expanding upon it in the following sections.

Figure 3 provides a schematic diagram of such a relay, where the angle $\mathrm{X}$ represents the field-of-view exclusion zone about the Sun as viewed from Earth, and angle Y is the equivalent spacecraft-Mars-Sun angle. 


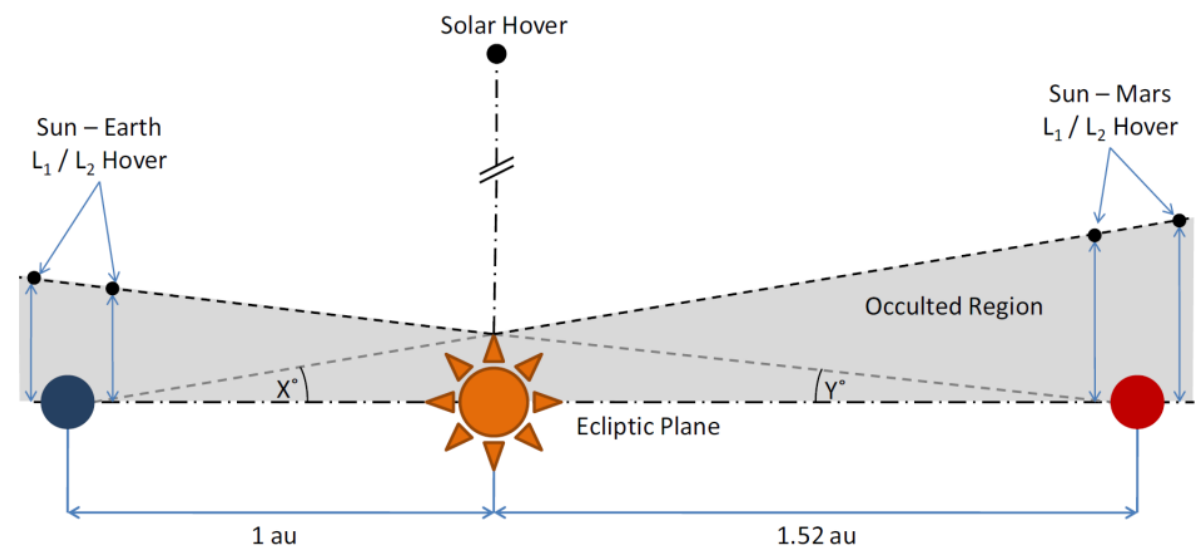

Fig. 3: Earth-Mars communication relay architecture options out of the orbital plane.

The technology requirements of such an array are largely determined by the field-of-view exclusion zone about the Sun, which is dependent on how close to the limb of the Sun radio signals can be transmitted without interference from the solar plasma. For design optimization purposes a spacecraft in proximity of Mars is preferred as the long slant range back to Earth can be compensated for through the use of a large Earth-based antenna.

If a 4-degree field-of-view exclusion of Mars from Earth is assumed (a realistic value if assuming communications in the X-band portion of the electromagnetic spectrum), the Sun - Mars stations can be determined to be located approximately $0.176 \mathrm{AU}$ out of the orbital plane, while the Sun - Earth stations can be determined to be located approximately $0.116 \mathrm{AU}$ outwith the orbital plane (since the equivalent spacecraft-Mars-Sun angle is then $2.64^{\circ}$ ). The much shallower gravitational potential well at Mars significantly increases the distance from the planet that a spacecraft can hover at in comparison to Earth. Naturally, different EM communication bands, such as Ka-band, define different field-of-view exclusion angles - this point will be returned to in the next subsection.

An interesting extension to this concept is to consider spacecraft in displaced orbits either leading or trailing the orbit of Mars, i.e. in the orbital plane. Considering the symmetry of Figure 3, the field-of-view exclusion defines a conic region around the Sun where Mars is hidden from the Earth. If this conic region is considered end-on from behind Mars, as shown in Figure 4, as well as achieving continuous communications by displacing a spacecraft directly above Mars, a spacecraft could also be displaced onto the circular (when projected in two dimensions) region around Mars defined by the field-of-view exclusion, so that one spacecraft was trailing and the other leading the orbit of Mars. 


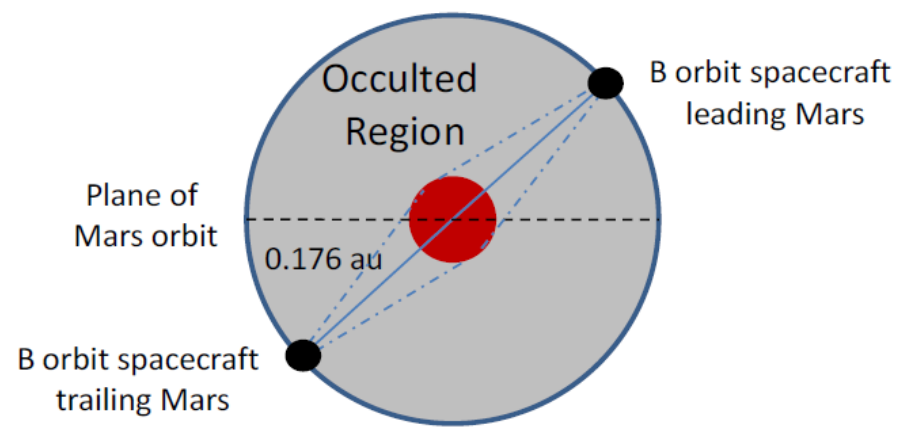

Figure 4: End-on view of an alternative Mars-Earth communication relay architecture option, looking along the orbital plane.

Naturally, as they track Mars they too will enter the blackout region: as depicted in Figure 4, the leading spacecraft will move beyond the edge of the blackout region as the trailing spacecraft moves into this region. However, the separation of the two spacecraft means that only one will ever be in this region at any given time, and, given the distance between the spacecraft, the arc of the orbit is easily sufficient to maintain a line-of-sight between the two spacecraft (i.e. one will not be occulted by Mars with respect to the other), allowing continual communications to still be achieved by relaying the signal from the occulted spacecraft to the one outside the occulted region and then on to Earth.

Positioning of the spacecraft then depends on what is required from the mission. The relay spacecraft will need less thrust to maintain their position with respect to Mars if they are not displaced out of the orbital plane, but that would only allow for communication with assets near the equator of the planet. To communicate with assets at higher latitudes it will be necessary to displace the spacecraft out of the orbital plane, which requires more thrust.

\subsection{Solar electric propulsion}

Having outlined the concept of the relay in the previous subsection, the amount of thrust required in order to occupy some potential hover points to enable the relay is quantified. To do this a spacecraft of total mass $1000 \mathrm{~kg}$ is assumed, with a solar electric propulsion (SEP) thruster with a maximum thrust of $300 \mathrm{mN}$ and a specific impulse ( $\left.\mathrm{I}_{\mathrm{SP}}\right)$ of 4500 seconds. These assumptions allow for a consideration of opportunities based on current or near-term SEP technology such as the Qinetiq T6 thruster, which will theoretically provide a thrust of up to $230 \mathrm{mN}$ at an $\mathrm{I}_{\mathrm{SP}}$ of above 4500 seconds for the BepiColombo mission [14], although such capabilities still have to be demonstrated within the context of any space mission.

Considering first of all the non-Keplerian orbit regions displaced out of the orbital plane, as illustrated in Figure 5, it can be seen that the $300 \mathrm{mN}$ thrust is sufficient to displace the spacecraft $0.176 \mathrm{AU}$ directly above Mars, which, from the previous section, is just enough to ensure that the spacecraft could communicate with Earth, assuming a 4 degree field-of-view exclusion, although clearly this is the bare minimum clearance required and thus leaves no margin. 


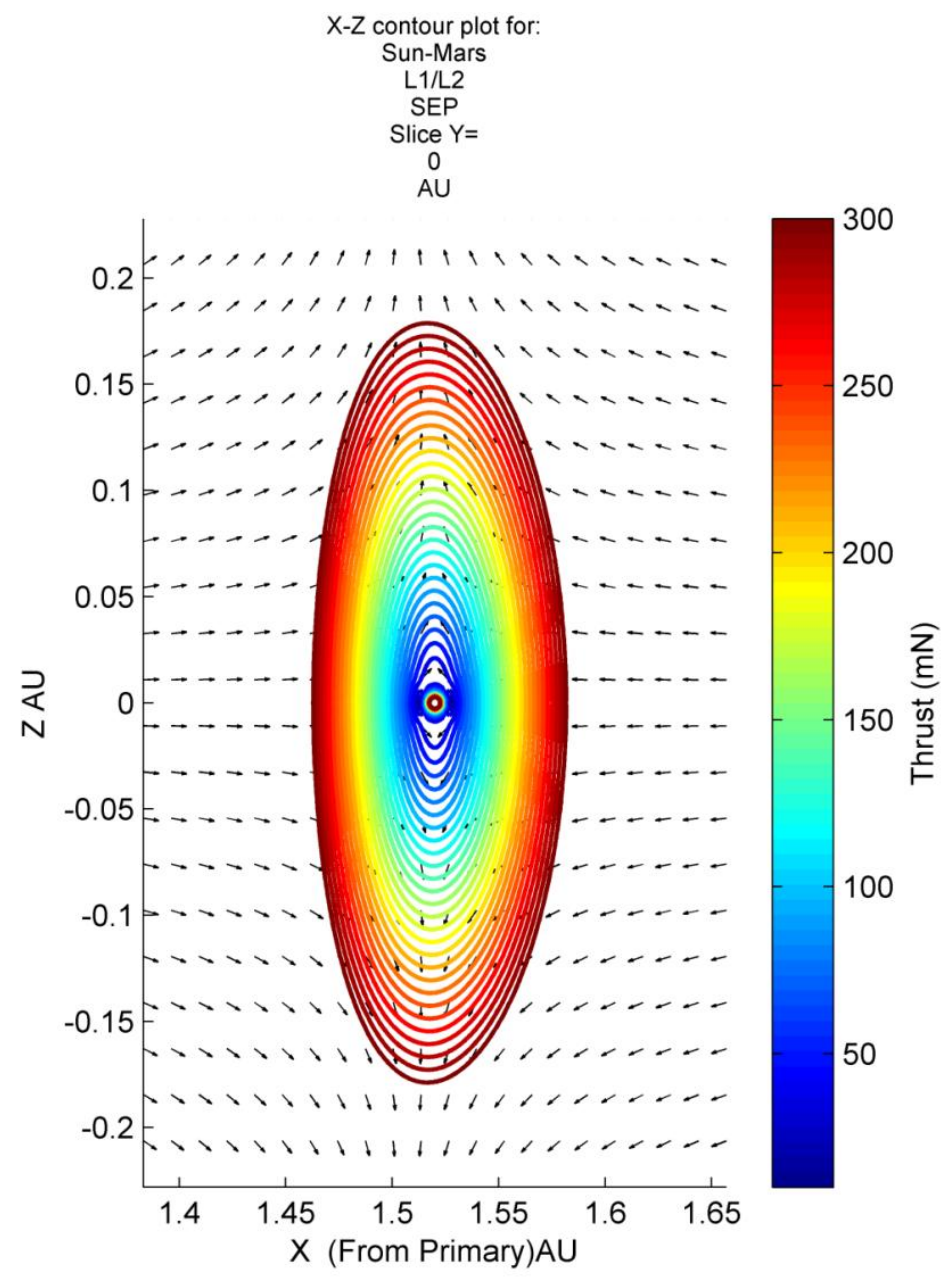

Figure 5: Non-Keplerian orbit contours at Mars in the plane displaced out of the orbital plane, for a $1000 \mathrm{~kg}$ solar electric propulsion (SEP) spacecraft with $300 \mathrm{mN}$ maximum thrust. The arrows indicate the required thrust orientation for the artificial equilibrium point.

However, there are some advantages to considering the dual spacecraft option discussed in the previous subsection, over the case of a single spacecraft hover. Firstly, hovering directly above Mars limits communications to just the polar region. If the spacecraft are trailing or leading the orbit then communication with the equatorial regions is enabled, with the ability to cover most of both hemispheres of Mars. A more important advantage can be shown by considering the equithrust contours in the plane illustrated by Figure 4, i.e. the $y-z$ plane, as shown in Figure 6 . 


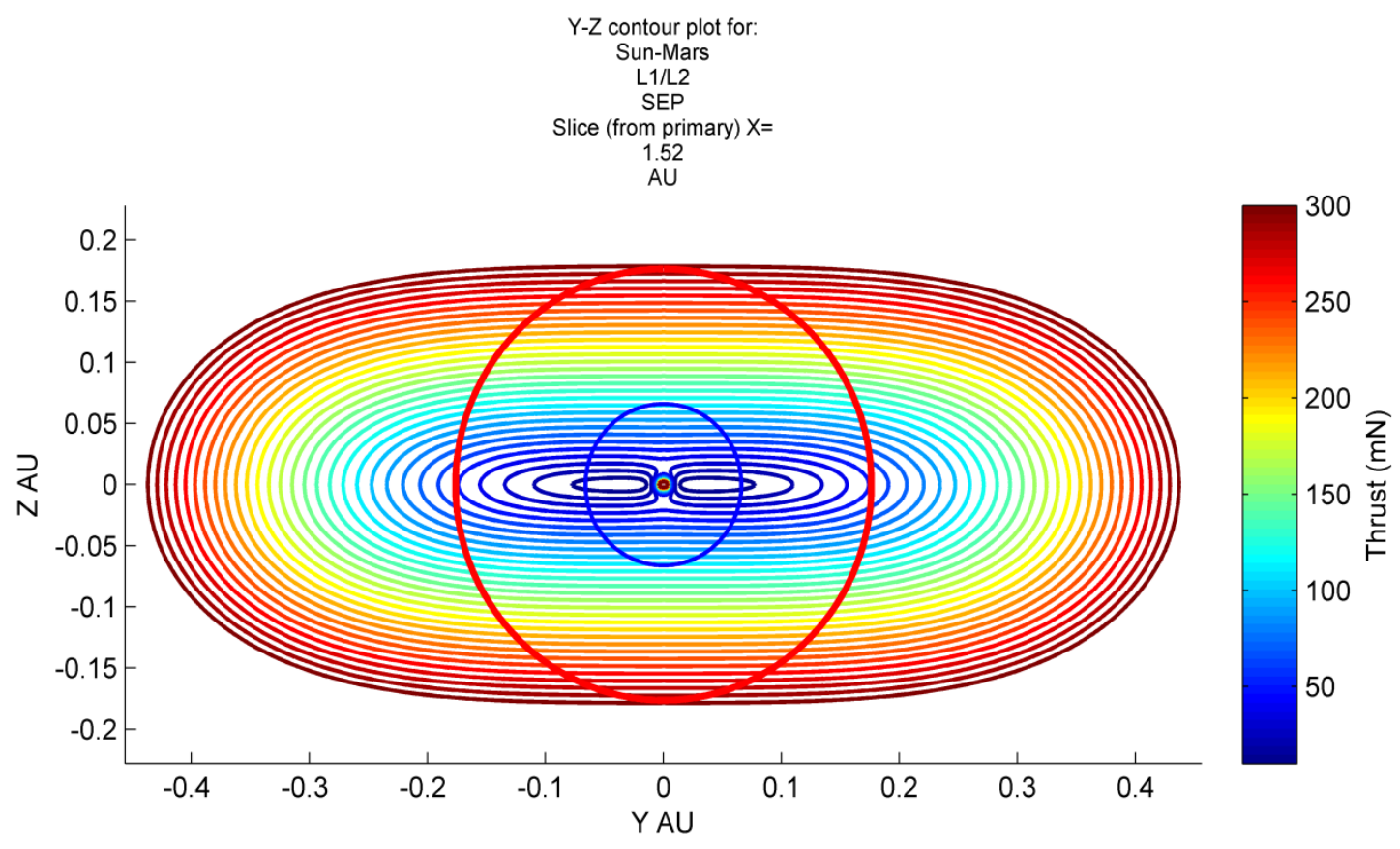

Figure 6: An end-on view of non-Keplerian orbits depicted by equithrust contours, for a 1000kg SEP spacecraft with 300mN maximum thrust about Mars, looking along the orbital plane. The red and blue circles indicate the field-of-view exclusion zone for $\mathrm{X}$-band and Ka-band communication respectively.

As can be seen it is much easier to displace the spacecraft orbit from Mars in this plane (i.e. along $y$ ) than out of it (i.e. along $z$ ) and so a spacecraft can occupy a non-Keplerian orbit on the surface defined by the field-of-view exclusion for less thrust if it trails or leads Mars rather than hovering directly above. For example, displacing the spacecraft 45 degrees out of the orbital plane of Mars would reduce the thrust requirements to approximately $200 \mathrm{mN}$, assuming the 4 degree field-of-view exclusion defined by X-band communication. So, practically, it may be more feasible to maintain the communications relay using two spacecraft with lower thrust than a single spacecraft which needs higher thrust.

This is true for the case of a 4 degree field of view exclusion of Mars from Earth by the Sun, which is a realistic value if it is assumed that the communication relay is via the $\mathrm{X}$-band portion of the electromagnetic spectrum. If instead the use of Ka-band is considered, the field of view exclusion can be reduced to just 1.5 degrees (as shown via the blue circle in Figure 6), with signals in this frequency range being less affected by the solar plasma. This obviously reduces the thrust requirements for the relay spacecraft considerably: it would then need to hover 9.872 million $\mathrm{km}(0.066 \mathrm{AU})$ directly above Mars, which could be done for a one tonne spacecraft with approximately $110 \mathrm{mN}$ of thrust, or there could be two spacecraft (one leading and one trailing the orbit of Mars, as in Figure 22) displaced 45 degrees out of the orbital plane (6.98 million km above Mars' orbital plane), which would require just $80 \mathrm{mN}$ of thrust each. Ka-band would be preferable for many other reasons, for example the higher bandwidth and thus data rate that comes with it, and the lower power requirements for the antenna, but Ka-band communication is technologically more challenging than that of $\mathrm{X}$-band and is thus more expensive and less readily-accessible as an 
option. However, NASA is already beginning the process of transitioning to Ka-band (because X-band is too narrow to support future high data rate missions), with the Mars Reconnaissance Orbiter being equipped with a fully functioning Ka-band communications suite [15].

One should also consider that the non-Keplerian orbit actually need only be maintained during periods of solar occultation, and hence it may be possible to extend the spacecraft lifetime by only using the thrusters to provide significant amounts of thrust during such periods and allowing the spacecraft to follow a conventional nearKeplerian orbit during other periods. For example, the synodic period of Mars (the temporal interval that it takes for an object to reappear at the same point in relation to two other objects) with respect to Earth and the Sun (and thus the occultation repeat period) is approximately 780 days, whereas the sidereal period (the temporal interval it takes an object to make one full orbit around the sun) is roughly 687 days. Thus one could envisage a mission that would see the SEP spacecraft thrusting to hover above Mars for 93 days to maintain communications whilst Mars is occulted, and then, when Mars is no longer occulted, using the thruster efficiently to re-acquire the relevant artificial equilibrium point (AEP) via a pre-planned orbital maneuver, returning to the correct point for the next occultation of Mars (where the thruster would be switched back on to occupy the non-Keplerian orbit position again). Thus the craft would only need to continually thrust at the levels outlined in the previous two paragraphs for about 90 days in every 2.13-year period (approximately) as opposed to the entire time, which would significantly extend the onstation time as allowed by the thruster propellant reserves. Consideration of such maneuvers is returned to in Section 4.2 .

Finally, it is worth considering the advantages of such a communications relay architecture option, over some of the more obvious potential setups. One might instead consider a relay consisting of a spacecraft at Earth's $\mathrm{L}_{4} / \mathrm{L}_{5}$ point, or likewise at Mars' $\mathrm{L}_{4} / \mathrm{L}_{5}$ point. The former case of an Earth-Earth $\mathrm{L}_{5}$-Mars relay then requires that a signal be sent over a total distance of approximately 3.21AU, with a distance of about 2.21 AU between Mars and the relay spacecraft. The latter case of an Earth-Mars $\mathrm{L}_{4}$-Mars relay spans a distance of approximately 3.73AU, with a distance between Mars and the relay space of around 1.52AU. Compare these numbers with the architecture as suggested above, with a relay spacecraft about 0.18AU from Mars and a total relay distance of around 2.7, meaning that the non-Keplerian orbit allows a relay station that spans a considerably smaller distance than using conventional Lagrange points, and enables a considerably higher data rate between the surface of Mars and the relay spacecraft.

\subsection{Hybrid Solar Electric Propulsion / Solar Sail}

Both SEP and solar sail low thrust propulsion systems have their own advantages and disadvantages. Solar sailing has the advantage of an effectively infinite specific impulse, i.e. requires no propellant/fuel, and thus can maintain continuous low thrust indefinitely (in theory, at least - in practice, long-term degradation of the optical surface may reduce the efficiency of the sail and propellant may be required for attitude control). However with SEP the thrust can be oriented in any direction, allowing access to artificial equilibria that a solar sail would be forbidden from with its inherent inability to thrust in the direction towards the Sun. Thus in theory there is a strong case for studying a 
device that would combine the best features of both systems, to obtain a hybrid sail: indeed, it has recently been suggested that such an approach may, in recognition of the high Advancement Degree of Difficulty of solar sailing, be the best means of advancing solar sail technology [16]. Such an idea has been considered in the literature previously - see, e.g. Leipold \& Götz [17] and Mengali \& Quarta [18], with the latter showing that hybrid sails have the attractive feature of reducing mission times for heliocentric transfers when compared to both the equivalent pure sail and pure SEP trajectories. Recently Baig \& McInnes [19], Simo \& McInnes [20] and Ceriotti \& McInnes [21] have all considered the case of displaced highly non-Keplerian orbits for the specific situation of a hybrid sail.

The analysis of Ref. [19] is followed by considering a partially reflecting hybrid sail consisting of an SEP thruster attached to the centre of a solar sail (as shown in Figure 7), a model adapted from that of Leipold \& Götz [17]. The solar sail is taken to be square, with part of the sail area at the centre of the sail covered by flexible thin film solar cells (TFSC), which act as a power source for the SEP system.

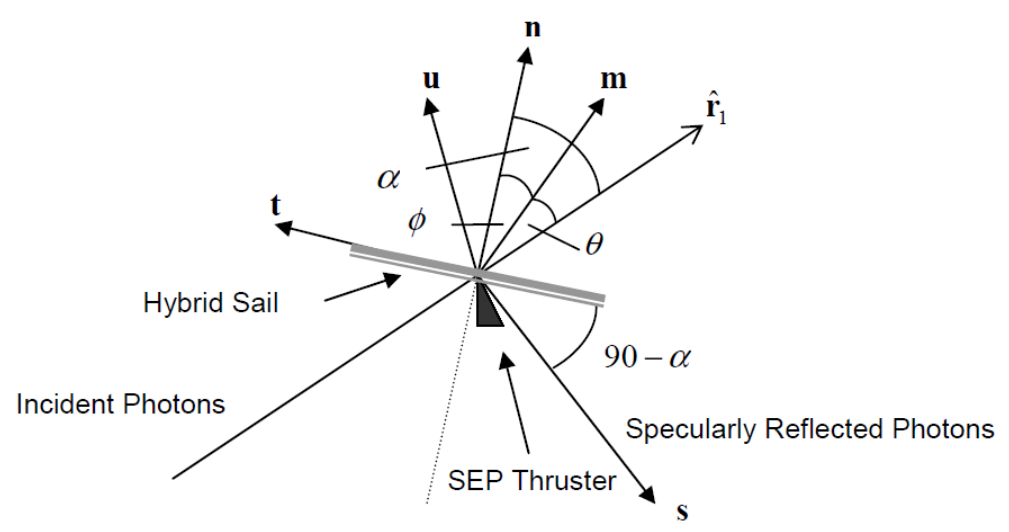

Fig. 7: Specularly reflecting hybrid sail solar radiation pressure force model.

In this model, $\boldsymbol{n}$ and $\boldsymbol{t}$ are the unit vectors normal to and transverse to the hybrid sail surface, respectively, $\hat{\boldsymbol{r}}_{1}$ is the direction of incident photons, and the unit vector $\mathbf{s}$ defines the direction of specularly-reflected photons. With such a model the total thrust of the hybrid sail due to both solar radiation pressure and solar electric propulsion is given by [19]

$$
\mathbf{F}_{T o t}=\mathbf{F}_{S E P}+\mathbf{F}_{\text {Sail }}=F_{n} \boldsymbol{n}+F_{t} \boldsymbol{t}+T \boldsymbol{u}
$$

where $F_{n}$ and $F_{t}$ denote the forces normal to and transverse to the sail respectively, and $T$ denotes the thrust from the SEP, where the unit vector $\boldsymbol{u}$ denotes the thrust direction. Expressions for $F_{n}$ and $F_{t}$ can be derived via consideration of the projected area of the hybrid sail in the direction of the incident photons, and shown to be, respectively,

$$
F_{n}=\left(\hat{\boldsymbol{r}}_{1} \cdot \boldsymbol{n}\right)^{2}\left[\left(1+\tilde{r}_{S}\right) P A_{S}+\left(1+\tilde{r}_{T F}\right) P A_{T F}\right]
$$


and

$$
F_{t}=\left(\hat{\boldsymbol{r}}_{1} \cdot \boldsymbol{n}\right)^{2}\left(\hat{\boldsymbol{r}}_{1} \cdot \boldsymbol{t}\right)\left[\left(1-\tilde{r}_{S}\right) P A_{S}+\left(1-\tilde{r}_{T F}\right) P A_{T F}\right]
$$

where $\tilde{r}_{S}$ and $A_{S}$ is the sail reflectivity and sail area, and $\tilde{r}_{T F}$ and $A_{T F}$ are the TFSC reflectivity and area.

Using Equation (8), expressions for both the nondimensional acceleration $\boldsymbol{a}_{s}$ due to the solar radiation pressure acting on the sail, and the nondimensional acceleration $\boldsymbol{a}_{S E P}$ due to the SEP thruster, can be derived by dividing by mass $m$ and dimensional reference acceleration $a_{r e f}=\omega^{2} R$ (where $R$ is the distance between the primary bodies) to give, respectively,

$$
\boldsymbol{a}_{s}=\boldsymbol{a}_{s} \boldsymbol{m}=\frac{1}{2} \beta_{0} \frac{m_{0}}{m} \frac{1-\mu}{r_{1}^{2}} g\left(\hat{\boldsymbol{r}}_{1} \cdot \boldsymbol{n}\right)^{2} \boldsymbol{n}+\frac{1}{2} \beta_{0} \frac{m_{0}}{m} \frac{1-\mu}{r_{1}^{2}} h\left(\hat{\boldsymbol{r}}_{1} \cdot \boldsymbol{n}\right)\left(\hat{\boldsymbol{r}}_{1} \cdot \boldsymbol{t}\right) \boldsymbol{t}
$$

and

$$
\boldsymbol{a}_{S E P}=\frac{T / m}{a_{r e f}} \boldsymbol{u}=a_{T} \boldsymbol{u} .
$$

Equation (11) has magnitude as determined by

$$
a_{s}=\frac{1}{2} \beta_{0} \frac{m_{0}}{m} \frac{1-\mu}{r_{1}^{2}} \sqrt{g^{2} \cos ^{2} \alpha+h^{2} \sin ^{2} \alpha} \cos \alpha
$$

where the parameters $g$ and $h$ are given by

$$
\begin{aligned}
& g=\left(1+\tilde{r}_{S}\right)-\frac{A_{T F}}{A_{T}}\left(\tilde{r}_{S}-\tilde{r}_{T F}\right) \\
& h=\left(1-\tilde{r}_{S}\right)+\frac{A_{T F}}{A_{T}}\left(\tilde{r}_{S}-\tilde{r}_{T F}\right)
\end{aligned}
$$

and $m_{0}$ is the initial mass of the hybrid sail, $\alpha$ is the sail pitch angle, and $\beta_{0} \equiv \sigma_{*} /\left(m_{0} / A_{T}\right)$ is the sail dimensionless lightness number, where $\sigma_{*}$ is the critical sail loading parameter of $1.53 \times 10^{-3} \mathrm{~kg} \mathrm{~m}^{-2}$.

Equations (11) and (12) therefore modify Equation (6) to give 


$$
\nabla V\left(\boldsymbol{r}_{\mathbf{0}}\right)=\boldsymbol{a}_{S}+\boldsymbol{a}_{S E P} \triangleq \boldsymbol{a}_{g c}
$$

i.e. $\nabla V\left(\boldsymbol{r}_{\mathbf{0}}\right)=\boldsymbol{a}_{g c}$ is the acceleration vector required to cancel the gravitational forces of the two primary masses and the centrifugal force in the rotating reference frame, a vector which can be achieved with a hybrid sail through the vector sum of the solar radiation pressure and SEP acceleration vectors.

The work of Ref. [19] is followed in defining a new reference frame that has its origin at the hybrid sail position and has a set of three orthogonal vectors $\left\{\boldsymbol{r}_{1}, \omega \times \boldsymbol{r}_{1}, \boldsymbol{r}_{1} \times\left(\omega \times \boldsymbol{r}_{1}\right)\right\}$. By making this frame transformation, the low thrust acceleration from the SEP system of the hybrid sail can be expressed in terms of the sail pitch and clock angles and $\alpha$ and $\delta$ respectively, giving

$$
a_{T}^{2}(\alpha, \delta)=a_{g c}^{2}-2 a_{g c} a_{s}(\cos \theta \cos \tilde{\theta}+\sin \theta \sin \tilde{\theta} \cos (\delta-\tilde{\delta}))+a_{s}^{2}
$$

where (see Ref. [19] for a fuller derivation) $\tilde{\theta}$ and $\tilde{\delta}$ are the cone and clock angles of the vector $\boldsymbol{a}_{g c}$ (and depend upon the artificial equilibrium point $\boldsymbol{r}_{0}$ ) and the cone angle of the hybrid sail is given by

$$
\tan \theta=\frac{(g-h) \tan \alpha}{g+h \tan ^{2} \alpha}
$$

It can then be shown that the hybrid sail clock angle should be aligned with the clock angle of the vector $\boldsymbol{a}_{g c}$ in order to obtain the maximum benefit from the solar sail [19], that is,

$$
\delta=\tilde{\delta}
$$

Considering the hybrid sail at time $t=0, m=m_{0}$ and from Equation (11) it is possible to orient the solar radiation pressure acceleration vector in order to obtain all or at least part of the acceleration vector $\boldsymbol{a}_{g c}$ required to occupy an artificial equilibrium point $\boldsymbol{r}_{0}$, since, for a pure solar sail or pure SEP system the required acceleration magnitude and thrust orientation are completely defined by the location of the artificial equilibrium point. Baig \& McInnes [19] proceed by selecting this optimum orientation to obtain the maximum benefit from the solar sail, and then use this acceleration to minimize the SEP thrust to achieve the desired vector $\boldsymbol{a}_{g c}$. Here, though, instead it is assumed that the thrust from the SEP system is fixed in the direction required for a given artificial equilibrium point, and added to that is the magnitude of the acceleration from the solar sail oriented to give the maximum benefit. This in effect opens up a greater volume of space that the hybrid sail can occupy over the pure SEP, pure sail, and Baig \& McInnes SEP thrust-minimized hybrid systems, thus allowing a determination if there are artificial equilibria that the hybrid spacecraft can access which would not be possible otherwise. This is because, in regions where the solar 
sail is effective, we have a greater magnitude of acceleration available for the hybrid solar sail/SEP spacecraft, which allows access to artificial equilibrium points that would otherwise be beyond the capabilities of the pure SEP system with thrust equal to that of the SEP part of the hybrid SEP/solar sail spacecraft.

With this in mind the analysis considered for a pure-SEP system, in determining the non-Keplerian orbit equithrust contours at Mars, can be repeated for the hybrid sail. Figure 8 shows the contours for orbits displaced out of the orbital plane for a hybrid spacecraft with a solar sail of characteristic acceleration $0.2 \mathrm{mms}^{-2}$ (equivalently, sail beta of 0.034 ) and sail reflectivity 0.9 . The sail area is $45 \mathrm{~m} \times 45 \mathrm{~m}=2025 \mathrm{~m}^{2}$, giving a sail loading of $45.63 \mathrm{gm}^{-2}$. The TFSC reflectivity is taken to be 0.4 and the TFSC area is assumed as $12 \mathrm{~m}^{2}$ purely for the sake of example. This sail is assumed to be attached to the usual 1000kg SEP-propelled spacecraft capable of a maximum thrust of $300 \mathrm{mN}$, as assumed previously. The pure-SEP contours of equivalent SEP thrust (i.e. not total acceleration/thrust) are overlaid in thin black lines, in order to compare the region of non-Keplerian orbits, and the arrows represent the direction of the vector $\boldsymbol{a}_{g c}$ required to occupy a given artificial equilibrium point.

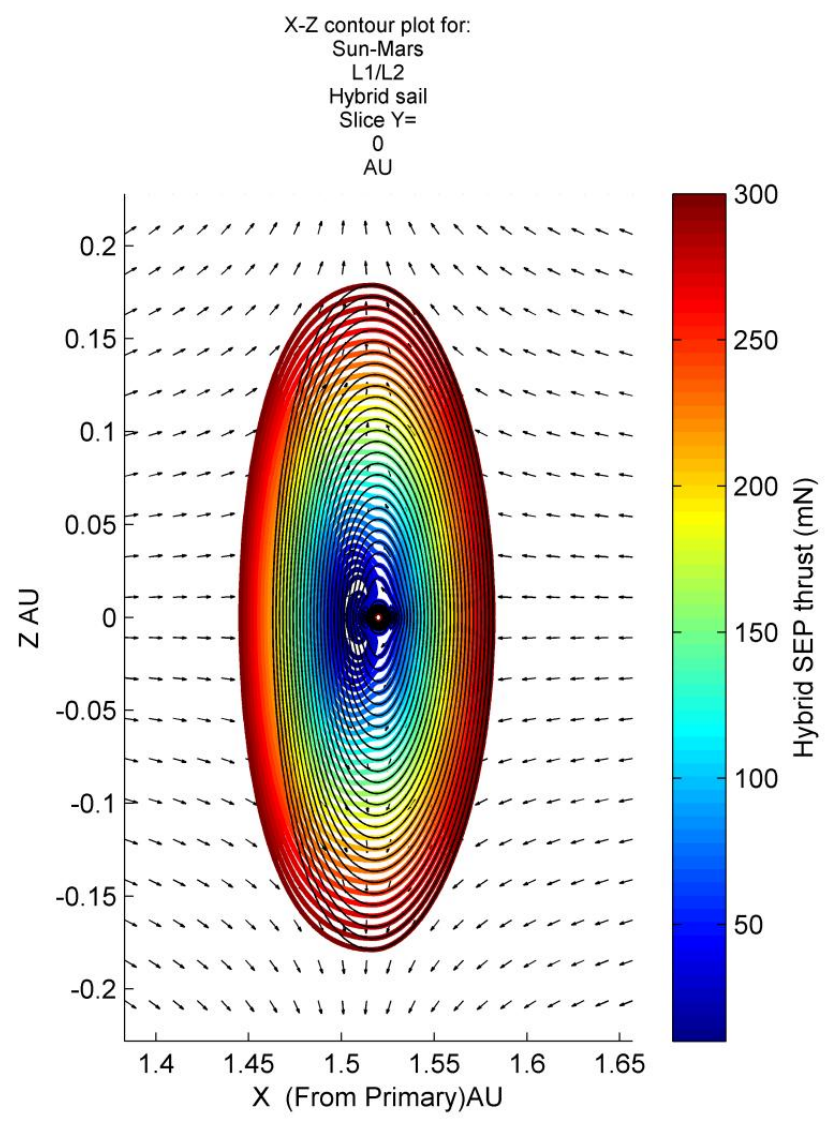

Fig. 8: Non-Keplerian orbit equithrust contours at Mars, projected onto the plane perpendicular to the orbital plane, for a hybrid sail with characteristic acceleration $0.2 \mathrm{mms}^{-2}$ and other parameters as given in the text. The black contours represent the contours for an equivalent pure-SEP system.

It is clear to see from Figure 8 that adding a solar sail to the SEP spacecraft allows access to a greater volume of space for non-Keplerian orbits: specifically, there is a reasonably large increase of available non-Keplerian orbits on 
the day-side of the planet around $\mathrm{L}_{1}$ and a small increase on the night-side of the planet around $\mathrm{L}_{2}$ (which is hard to see given the scale of this particular plot but will be more obvious in subsequent plots, particularly Figures 12 and 13). This non-symmetry is to be expected, given the regions of non-Keplerian orbits the pure sail can access. It is also clear that the addition of the solar sail does not allow the hybrid to be displaced any further out of the orbital plane for the same amount of thrust, and thus in the context of the Earth-Mars communication relay as described previously this is perhaps a disappointing result. However, deeper consideration of the solar sail reveals why both of these points are indeed the case.

Firstly, consider the artificial equilibrium solutions for the solar sail itself. McInnes [22] considered the equilibrium solutions for a non-perfect solar sail, which is of importance because, aside from reducing the magnitude of the solar radiation pressure exerted on the sail surface, the partial reflectivity and hence finite absorption means that the radiation pressure force vector is no longer normal to the sail surface, and thus the equilibrium surfaces and accessible regions are slightly different, as compared in Figure 9.
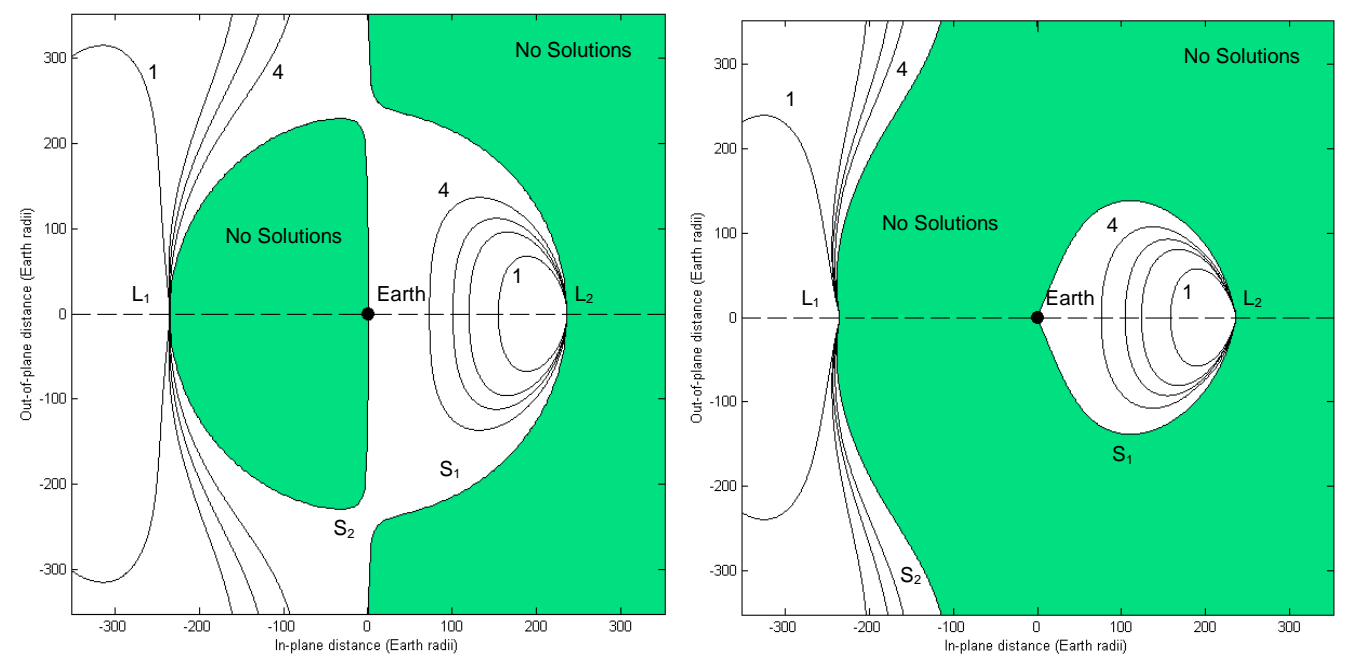

Fig. 9: Comparison of contours of sail loading in the x-z plane with reflectivity $\eta=1$ (left) and $\eta=0.9$ (right), where the contours are, in $\mathrm{gm}^{-2}:$ [1] 30 [2] 15 [3] 10 [4] 5.

For a non-perfect reflectivity, it can be seen that there are no regions of possible solution directly above the planet (in this instance, the Earth, but the principle is the same for Mars). Thus it is impossible to have a solar sail stationed at a hover point directly above the planet. Even regardless of the reflectivity it can be seen that this is effectively the case: for a perfectly reflecting sail there is a region of singularity above the Earth where a sail would have to have effectively infinite acceleration in order to occupy such a spot. Bearing Figure 9 in mind it is not surprising, therefore, that the hybrid sail shows no advantage over the pure SEP system for non-Keplerian orbits displaced directly above the planet, as in Figure 8. 
Regardless, the fact that there is no advantage in using a hybrid to hover directly above Mars does not in itself rule out the possibility of a hybrid system being potentially more useful than SEP alone as part of such a communications relay. Hovering directly above Mars is not exactly the same as a polesitter spacecraft, i.e. a spacecraft constantly aligned with the polar axis of the Earth, because of the tilt of that axis. Replotting Figure 8 to illustrate the $\pm 25.2^{\circ}$ axial tilt of Mars, as shown in Figure 10, four specific points are highlighted in purple which illustrate where the spacecraft can be stationed such that they are directly above the pole of Mars. Three of these points are on the dayside of Mars, with coordinates of $(1.462,0.125),(1.472,0.103)$ and $(1.490,0.065)$ respectively, in AU. The first two points show that the addition of the solar sail component of the hybrid extends the distance the spacecraft can hover directly above the pole at the summer solstice from 0.114AU to 0.137AU (i.e. can now station at the former point as opposed to the latter), or, equivalently, to occupy the latter point the hybrid spacecraft needs a thrust of only $240 \mathrm{mN}$ from the SEP component, compared to the $300 \mathrm{mN}$ required for a pure-SEP spacecraft. The third point shows the minimum distance required to complete the communications relay in Ka-band, which requires approximately $130 \mathrm{mN}$ from the hybrid SEP and $190 \mathrm{mN}$ from the pure-SEP. The fourth point on the night-side of the planet shows that having the sail is of no additional benefit here.

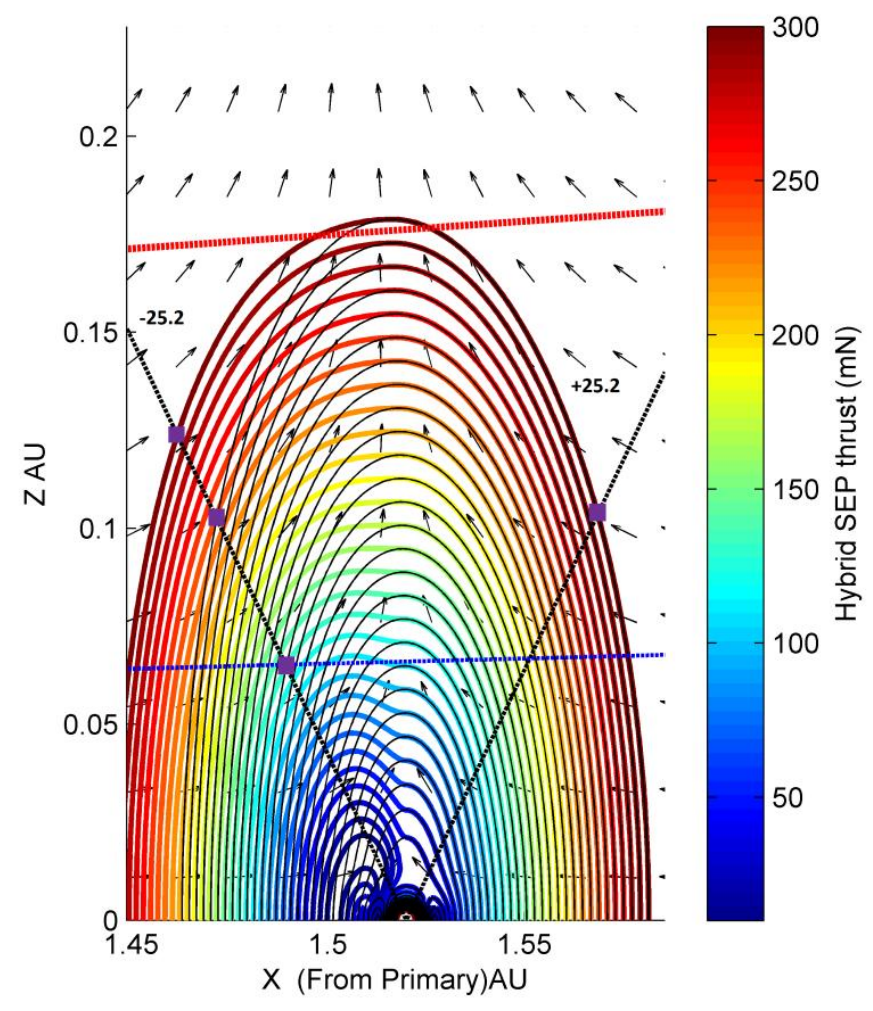

Fig. 10: Non-Keplerian orbit equithrust contours at Mars, projected onto the plane perpendicular to the orbital plane, for the hybrid sail as plotted in Figure 8. The black contours represent the contours for an equivalent pure-SEP system, and $-/+25.2^{\circ}$ are the angles of the polar axis of Mars, denoted by dashed lines, with respect to the normal to the orbital plane at the summer and winter solstices respectively. The red and blue dashed lines represent the field-of-view exclusion angle of $X$ and Ka-bands respectively. 
Thus it can be seen that the addition of the sail can reduce the SEP requirements to hover directly above the pole of Mars at the summer solstice, or, equivalently, allow the spacecraft to hover higher than previously possible without the sail. Clearly, in this case Figure 10 shows that it would only make sense to do this over the day-side of the planet, with this region being where the sail is most effective: the volume of space within which equilibria are possible on the night-side of the Earth is severely constrained with a realistic solar sail, as shown in Figure 9. This argument is effectively analogous to that made by Ceriotti and McInnes, who determined families of optimal periodic polesitter orbits above Earth that minimized the SEP propellant consumption over a 1-year period [21], and showed that these optimal orbits are displaced less far out of the orbital plane when on the night-side of Earth.

The enhanced polesitter opportunities at Mars with hybrid propulsion in turn translate into a partial benefit of using hybrid propulsion for a communications relay at Mars. From Figure 10, it can be seen that while the hybrid spacecraft can be displaced further from the pole of Mars, it can still only be displaced 0.125AU out of the orbital plane, which is sufficient to complete the relay for the 1.5 degree field-of-view exclusion angle implied by Ka-band communication (represented by the dashed blue line in Fig. 10) but not for the 4 degree angle of X-band communication (the dashed red line in Fig. 10). Therefore, considering the region between the red and blue lines, on the day-side of Mars, it can be seen there is an area where the addition of a small and technically feasible near-term solar sail to an SEP component has some ability to reduce the thrust required from the SEP component, compared to the pure-SEP equivalent spacecraft. Hence for the specific case of a Ka-band Earth-Mars communication relay, communicating with an asset on the day-side of Mars in the approximate vicinity of the poles, hybrid propulsion could be an advantage to the mission. However, if the ground assets are located away from the poles then hybrid propulsion proves less advantageous (although this only considers the case of one relay spacecraft, and not two i.e. the case of Figure 4), and if the assets are stationed on the night-side of Mars, then hybrid propulsion provides no advantage. This is also true if the assets are stationed on the day-side during Mars' northern hemisphere winter, since it is important to remember that the poles will rotate, i.e. between northern hemisphere summer and winter the polar axis sweeps out a cone, and hence the benefit of the sail is only felt during the summer.

Utilizing hybrid propulsion in a relay with X-band communication is a bit more complicated, at least for the case of a polesitter. This is essentially due to the second failing of the sail component, namely that of solar flux - in that being so far from the Sun (1.52 AU), the flux of radiation striking the sail and providing the photon pressure is considerably less than that of at, say, Earth. The characteristic acceleration of $0.2 \mathrm{mms}^{-2}$, which is a reasonable nearterm goal to aim for in solar sail construction, only translates into an actual maximum acceleration of approximately $0.087 \mathrm{mms}^{-2}$ at Mars. This is only a small fraction of the $0.3 \mathrm{mms}^{-2}$ being considered for a 1-tonne SEP spacecraft with $300 \mathrm{mN}$ of maximum thrust, and is obviously compounded by the solar sail acceleration being a function of the pitch angle, with its $\cos ^{2} \alpha$ dependence reducing that still further as the sail is moved out of the orbital plane. Therefore it is clear that it will require a significantly advanced solar sail in order to provide sufficient acceleration at Mars to either reasonably reduce the burden on the SEP thruster to maintain a given hover or to hover further above the pure SEP spacecraft. Figure 11 illustrates the hybrid equithrust contours with the same parameters as 
before but with a solar sail of characteristic acceleration of $1 \mathrm{mms}^{-2}$, five times greater than that suggested as realistic in the near-term. Again the equivalent thrust contours of the pure SEP system are displayed in thin black lines and the field-of-view exclusion of $\mathrm{X}$ and Ka-band communication is represented by red and blue dashed lines respectively.

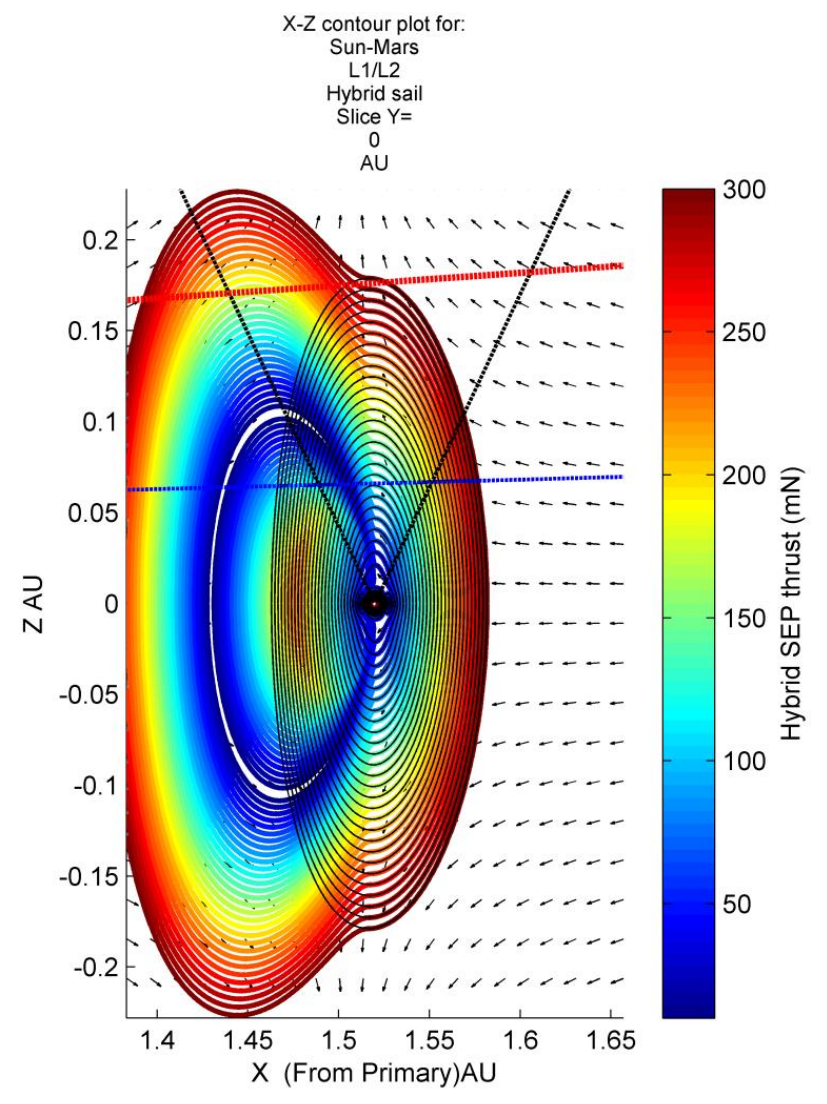

Fig. 11: Non-Keplerian orbit equithrust contours at Mars, projected onto the plane perpendicular to the orbital plane, for the hybrid solar sail with sail characteristic acceleration $1 \mathrm{~mm} \mathrm{~s} \mathrm{~s}^{-2}$. The black contours represent the contours for an equivalent pure-SEP system. The red and blue dashed lines represent the fieldof-view exclusion angle of $X$ and Ka-bands respectively.

It can be seen from Figure 11 how, with this much greater performance hybrid sail, a relay spacecraft could hover at greater distances above Mars if it was displaced closer to or further from the Sun, with it being possible to station up to $0.226 \mathrm{AU}$ above Mars (although not directly) as opposed to 0.176 AU for the pure-SEP system. Equivalently the relay spacecraft could hover at the same distance of $0.176 \mathrm{AU}$ out of the orbital plane, except displaced 0.07AU closer to the Sun - with this performance of sail, the SEP thrust needed is just $184 \mathrm{mN}$, as opposed to a pure-SEP system of $300 \mathrm{mN}$. The minimum amount of thrust required from the SEP component with this solar sail to have a Martian polesitter that would be able to complete the relay in X-band communication would be approximately $180 \mathrm{mN}$ - this would require approximately $500 \mathrm{mN}$ with SEP alone. In this case perhaps the best trade-off would be to have a hybrid with solar sail of characteristic acceleration of $0.6 \mathrm{mms}^{-2}$ and SEP maximum thrust of $300 \mathrm{mN}$, which would just allow a polesitter in X-band to complete the relay. 
Clearly therefore there is a complex balance to be achieved in designing such a communications relay, with regards to the design of the relay spacecraft. The main factors are where the ground assets to be communicated with are stationed on the Martian surface and which communications band the relay spacecraft will communicate with Earth in. Both factors determine how far out of the orbital plane the spacecraft needs to be. To summarise the previous results, in Ka-band a Martian polesitter needs a minimum of $190 \mathrm{mN}$ from pure SEP and $130 \mathrm{mN}$ plus a solar sail of characteristic acceleration of $0.2 \mathrm{mms}^{-2}$. For X-band, the polesitter needs a minimum of $500 \mathrm{mN}$ from pure SEP, $300 \mathrm{mN}$ when a solar sail of characteristic acceleration of $0.6 \mathrm{mms}^{-2}$ is included and $180 \mathrm{mN}$ when a solar sail of characteristic acceleration of $1 \mathrm{mms}^{-2}$ is included. Hence any such mission will involve a trade-off, with the design of the relay driving the technology requirements of the spacecraft. In some cases it may be that the required solar sail performance is much greater than currently envisaged, and only produces comparatively modest reductions in required thrust from the SEP component, and that therefore the technological "leap" to develop the proven SEP technology to produce more thrust may be considerably smaller than the equivalent "leap" of taking current SEP technology and adding a solar sail that is still well beyond realistic near-term capability. In some cases a more advanced solar sail may provide very large reductions in the required SEP thrust, and thus make the development of the sail worthwhile. Even if the desired sail is too difficult to achieve, pushing the boundaries of the sail technology will reduce the need to do the same on the SEP side. In other cases it may be that the inclusion of a small solar sail is worth the effort because it produces enough reduction in thrust from the SEP so as to significantly extend the propellant reserves and thus lifetime of the thruster. In yet other cases it will be true that the addition of a solar sail provides little-to-no benefit, regardless of sail parameters - i.e., where the polesitter requirement is relaxed. Such considerations would need to be addressed when specifying the objectives of the communication relay, and further complexities will arise if considering the architecture options with a second relay spacecraft as described by Figure 4.

It should be remembered here that the analysis only assumes the case of a large SEP system and small solar sail. However, what is evident is that for this particular combination the hybrid sail is most effective in the orbital plane, where, given the direction of the solar radiation pressure, the sail can be oriented to provide exactly the same component of acceleration $\boldsymbol{a}_{g c}$ as the equivalent performance SEP thruster. This fact may be of greater use in other potential non-Keplerian orbit missions, especially for those closer to the Sun where the photon pressure is higher.

One such example would be that of the GeoStorm mission concept, which aims to improve the warning time of impending space weather events, such as geomagnetic storms, via the application of emerging new technologies such as solar sails and micro-spacecraft. Currently, probes orbiting the Earth-Sun $\mathrm{L}_{1}$ point can provide approximately 30 minutes advance warning of an approaching CME. The 1999 ST-5 GeoStorm mission proposal suggested the use a solar sail of characteristic acceleration $0.169 \mathrm{mms}^{-2}$ to access an artificial displaced orbit at a point sunward of $\mathrm{L}_{1}$ (0.993AU from the Sun), instead maintaining station at 0.984AU [6]. This would increase the warning time of an approaching magnetic storm by a factor of approximately 3. McKay et al. $[10,13]$ applied the same principle to a continuous low-thrust SEP spacecraft of $m=1000 \mathrm{~kg}$, showing that $300 \mathrm{mN}$ of thrust would 
make it possible to station at approximately 0.981AU, i.e. further away from Earth than the ST-5 proposal, although not by much given the extra mass required due to the propellant required to station there. Nevertheless, this would allow for a geomagnetic storm warning time of upwards of 90 minutes.

However, considering a hybrid sail with the same parameters as that of the sail in Figure 8, it can be seen that this warning time can be further improved, as illustrated by Figure 12. It would be possible to station the spacecraft at 0.971AU, which would increase the warning time yet further by a factor of about 2 compared to the pure-SEP station point of $0.981 \mathrm{AU}$, or a total of a factor of about 6 times as much warning over spacecraft stationed at $\mathrm{L}_{1}$. Equivalently, one could station the hybrid at the $0.981 \mathrm{AU}$ point, which would reduce the thrust required from the SEP system from $300 \mathrm{mN}$ to about $100 \mathrm{mN}$. This would also extend the life of the mission from the 3 years estimated in Ref. [10] to about 8.5 years, and would come with the added benefit that when the SEP propellant ran out, the solar sail would still produce some acceleration and thus equilibriate to a new non-Keplerian orbit. With just the sail acceleration the hybrid would station at around $0.985 \mathrm{AU}$, which is still an improvement in storm warning time over spacecraft stationed at $\mathrm{L}_{1}$, and would allow the spacecraft to be kept in deep space - potentially allowing for a refueling mission before returning to the original station point. In theory the solar sail could keep the hybrid at this "backup" station for an infinite amount of time, although in practice degradation of the reflective surface and onboard electronics would eventually terminate the mission.

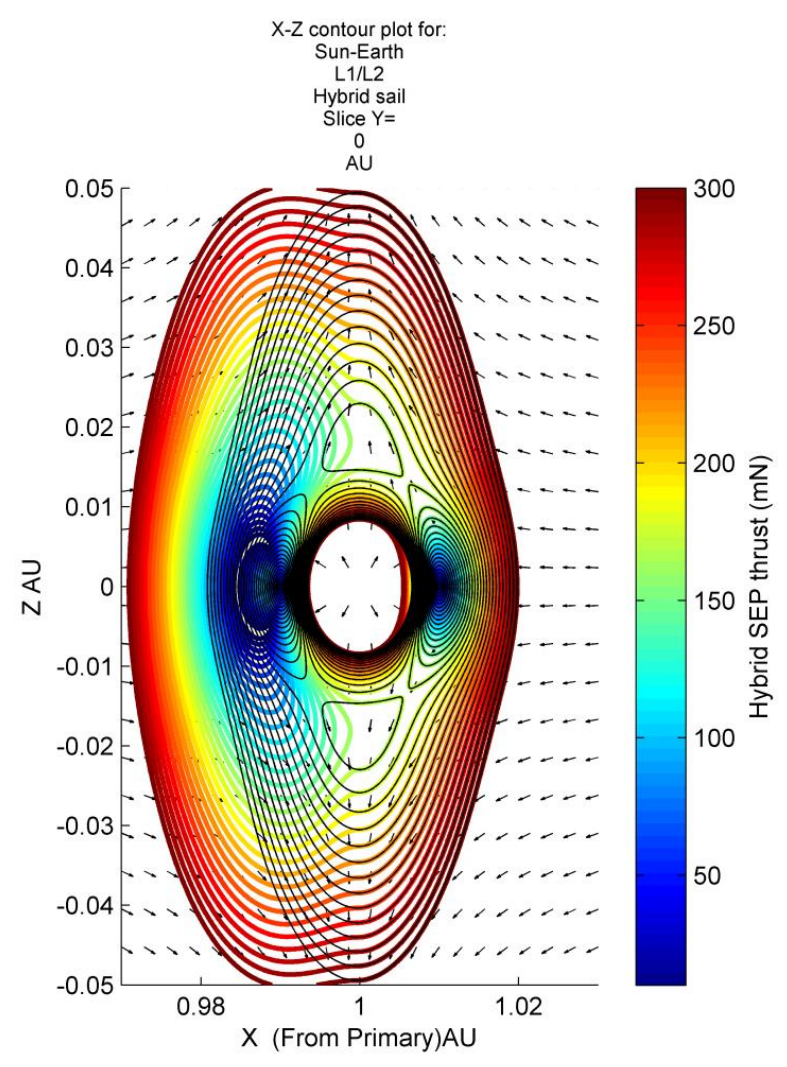

Fig. 12: Non-Keplerian orbit equithrust contours at Earth, projected onto the plane perpendicular to the orbital plane, for a hybrid sail with characteristic acceleration $0.2 \mathrm{mms}^{-2}$ and other parameters as given in the text. The black contours represent the contours for an equivalent pure-SEP system. 
Of course, it is important to remember here that although equithrust surfaces are considered, no propulsion system actually delivers an equal thrust throughout the lifetime of the spacecraft, due to either depletion of reaction-mass or, in the case of solar sailing, the degradation of the optical surface [23]. As such, the propulsion system would have to be throttled to adjust for either the increasing (for depletion of reaction-mass) or decreasing (for degradation of the optical surface) acceleration vector magnitude.

As discussed previously, further advantages of the hybrid sail are made even clearer when considering the nonKeplerian orbit contours at celestial bodies even closer to the Sun than Earth: for example alone, those of Venus are displayed in Figure 13. This should be borne in mind when other missions are being considered, such as the solar sail mission concept for a Mercury Sun-Synchronous orbiter [24]; however such discussion is not included here.

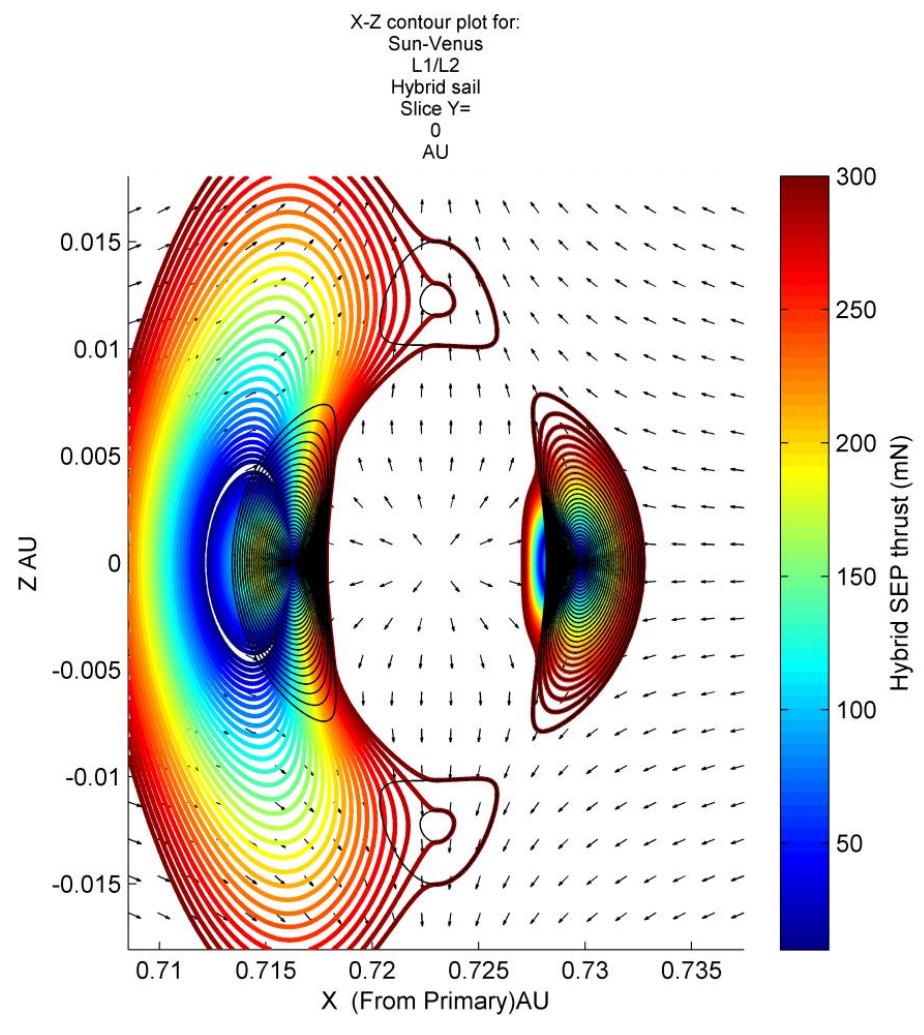

Fig. 13: Non-Keplerian orbit equithrust contours at Venus, projected onto the plane perpendicular to the orbital plane, for a hybrid sail with characteristic acceleration $0.2 \mathrm{mms}^{-2}$ and other parameters as given in the text. The black contours represent the contours for an equivalent pure-SEP system.

\section{$\underline{4-\text { Mission Analysis }}$}

\subsection{Trajectory Insertion}

Previously it was determined that the hybrid option does bring some advantages, in terms of stationing relay spacecraft above Mars for the purposes of a communications relay, for specific architecture options. However, for simplicity and generality, this section focuses only on mission analysis and contingency cases for the pure-SEP 
option. For reference, all the calculations in this section were performed with the software tool DITAN (Direct Interplanetary Trajectory Analysis) [25]. DITAN implements a direct transcription method based on Finite Elements in Time developed on a spectral basis [26, 27].

The analysis proceeded by determining the possibilities to insert a spacecraft into position, to enable such a relay. Initial analysis focused on the simple case of insertion trajectories to the point $0.176 \mathrm{AU}$ directly above Mars, with both chemical and low-thrust transfers being studied. The basis behind the transfers was to assume a low thrust transfer from LEO (of 500km altitude) using, in the case of SEP missions, a thruster which has a maximum thrust of $300 \mathrm{mN}$ and a specific impulse of $4500 \mathrm{sec}$, on a spacecraft of total mass $1000 \mathrm{~kg}, 500 \mathrm{~kg}$ of which is propellant, although as will be seen for optimisation of some of the scenarios some of these parameters were treated with a degree of flexibility. The transfers were optimized such that the propellant consumption was minimized.

For the chemical option a direct bi-impulsive Earth-Mars transfer with intermediate change plane maneuver was considered, as was an Earth-Earth-Mars (EEM) transfer with a resonant swing-by of the Earth to reduce the departure excess velocity at the Earth. The results of these options are displayed in Table 1.

Table 1: Chemical option for the Mars Communication Relay Mission

\begin{tabular}{|l|c|c|c|c|}
\hline & $\begin{array}{c}\text { Specific Impulse } \\
\text { Isp }(\mathbf{s})\end{array}$ & $\begin{array}{c}\text { Propellant } \\
\text { consumed } \\
\mathbf{m}_{\mathrm{p}}(\mathbf{k g})\end{array}$ & $\begin{array}{c}\text { Time of Flight } \\
(\text { days })\end{array}$ & $\mathrm{v}_{\text {inf }}(\mathbf{k m} / \mathbf{s})$ \\
\hline $\begin{array}{l}\text { Two-Impulse } \\
\text { Transfer }\end{array}$ & 315 & 660.69 & 808.41 & 3.05 \\
\hline $\begin{array}{l}\text { EEM } \\
\text { Transfer }\end{array}$ & 315 & 714.63 & 1230.0 & 0.9 \\
\hline
\end{tabular}

From the results in Table 2 the chemical option appears to be infeasible, given the propellant consumption required. The plane change maneuver is excessively high and Mars gravity does not sufficiently help during the capture maneuver. Even the use of a single Earth resonant flyby does not solve the problem, because it does not provide the necessary $\Delta v$. In the calculation of the propellant mass the departure excess velocity $\mathrm{v}_{\text {inf }}$ is not included - this is why the EEM trajectory presents a higher propellant consumption. Adding more flybys and increasing the excess velocity at the Earth would improve the propellant consumption, but it would still remain very high.

For the low-thrust options a direct transfer departing with zero excess velocity and an Earth-Earth-Mars transfer with resonant flyby of the Earth were both considered assuming both constant and variable thrust possibilities, with the results of these transfers shown in Table 2. The low-thrust options appear to be more feasible than the chemical options discussed previously, in terms of propellant consumption. 
Table 2: Low-thrust option for the Mars Communication Relay Mission

\begin{tabular}{|l|c|c|c|c|}
\hline & $\begin{array}{c}\text { Specific Impulse } \\
\text { Isp }(\mathbf{s})\end{array}$ & $\begin{array}{c}\text { Propellant } \\
\text { consumed } \\
\mathbf{m}_{\mathrm{p}}(\mathbf{k g})\end{array}$ & $\begin{array}{c}\text { Time of Flight } \\
(\mathbf{d a y s})\end{array}$ & $\mathbf{v}_{\text {inf }}(\mathbf{k m} / \mathbf{s})$ \\
\hline $\begin{array}{l}\text { Direct } \\
\text { Variable Thrust }\end{array}$ & $\mathbf{3 0 0 0}$ & $\mathbf{1 9 2 . 0}$ & $\mathbf{1 2 5 2 . 7 3}$ & $\mathbf{0 . 0}$ \\
\cline { 2 - 5 } $\begin{array}{l}\text { Direct } \\
\text { Constant Thrust }\end{array}$ & $\mathbf{4 5 0 0}$ & $\mathbf{1 1 5 . 0}$ & $\mathbf{1 1 8 6 . 0}$ & $\mathbf{0 . 0}$ \\
\hline $\begin{array}{l}\text { EEM } \\
\text { Variable Thrust }\end{array}$ & $\mathbf{3 0 0 0}$ & $\mathbf{2 0 3 . 0}$ & $\mathbf{4 3 8 . 7 3}$ & $\mathbf{0 . 0}$ \\
\hline $\begin{array}{l}\text { EEM } \\
\text { Constant Thrust }\end{array}$ & 4500 & $\mathbf{9 7 . 0 8}$ & 173.18 & 0.9 \\
\hline
\end{tabular}

However, one must take into account that the options with variable thrust consider a simple engine model in which the power delivered by the solar arrays is proportional to $1 / r_{s}^{2}$ where $r_{s}$ is the distance from the Sun in AU. The low-thrust options thus present a small systems engineering issue - in particular the ones with high specific impulse. This is because, if a standard ion engine with a typical specific power of $27-30 \mathrm{~W} / \mathrm{mN}$ is considered, the power system would need to deliver between 8 and $9 \mathrm{~kW}$. If a solar array efficiency of 0.25 at 1AU (BOL) is assumed, with an inherent degradation of 0.8 , and a 0.7 reduction due to end-of-life degradation, then $8-9 \mathrm{~kW}$ correspond to an area of the solar arrays between 42 and $47 \mathrm{~m}^{2}$ only for the propulsion system and without margins.

It is thus required that the thrust level and/or the Isp are substantially lowered, in order to reduce the solar array area to a more realistic figure. If one instead assumes a thruster capable of producing a reduced thrust level of $220 \mathrm{mN}$ at a distance of $1 \mathrm{AU}$, namely an Astrium RIT-XT engine operating at $3000 \mathrm{~s}$ with a $22 \mathrm{~W} / \mathrm{mN}$ of specific power, then the required area of the solar arrays would be just $25.3 \mathrm{~m}^{2}$, for the propulsion system only (with no margin, and $25 \%$ efficiency of the cells). If a 500W total subsystem power requirement is considered then that area increases to $39.1 \mathrm{~m}^{2}$, if a $20 \%$ margin is also included. For comparison, consider the Rosetta and SMART-1 missions - the former had a solar array area of $61.5 \mathrm{~m}^{2}$ for (approximately) a $3000 \mathrm{~kg}$ spacecraft [28], and the latter had a solar array area of about $10 \mathrm{~m}^{2}$ for (approximately) a $370 \mathrm{~kg}$ spacecraft [29]. Thus the spacecraft for an artificial equilibrium point relay mission at Mars would be somewhere in between Rosetta and SMART-1's requirements.

If the thruster could indeed produce $220 \mathrm{mN}$ thrust at $1 \mathrm{AU}$, that would correspond to $80 \mathrm{mN}$ at the aphelion of Mars, which is, as discussed in Section 2, the thrust level required for the Mars relay architecture option featuring two spacecraft each displaced 45 degrees out of the orbital plane and utilising Ka-band radio communication. Thus trajectory insertion to this point is now considered. In order to have $1000 \mathrm{~kg}$ wet mass at Mars the trajectory was reoptimized, minimizing the initial mass rather than the final one. The results are summarized in Table 3 for both the direct and the EEM transfer options, and the trajectories are displayed in Figures 14 and 15 respectively for the direct and EEM transfers. The respective thrust profiles of these trajectories are displayed in Figure 16. Table 3 shows how the EEM transfer option may be preferable because, although it takes twice as long as the direct option, 
it requires less propellant and takes less life out of the thruster to do so, needing only 8200 hours of thrust against nearly 12000 hours for the direction option.

Table 3: Low-thrust option for the Mars Communication Relay Mission: reduced thrust case

\begin{tabular}{|l|c|c|c|c|c|c|}
\hline & $\begin{array}{c}\text { Specific } \\
\text { Impulse } \\
\text { Isp (s) }\end{array}$ & $\begin{array}{c}\text { Initial mass } \\
(\mathbf{k g})\end{array}$ & $\begin{array}{c}\text { Propellant } \\
\text { consumed } \\
\mathbf{m}_{\mathrm{p}}(\mathbf{k g})\end{array}$ & $\begin{array}{c}\text { Time of } \\
\text { Flight (days) }\end{array}$ & $\mathbf{v}_{\text {inf }}(\mathbf{k m} / \mathbf{s})$ & $\begin{array}{c}\text { Thrust } \\
\text { Time (h) }\end{array}$ \\
\hline $\begin{array}{l}\text { Direct } \\
\text { Variable } \\
\text { Thrust }\end{array}$ & 3000 & 1220.07 & 220.07 & 621.1 & 0.0 & 11985.0 \\
\hline $\begin{array}{l}\text { EEM } \\
\text { Variable } \\
\text { Thrust }\end{array}$ & 3000 & 1131.63 & 131.63 & 1164.22 & 0.9 & 8196.2 \\
\hline
\end{tabular}

Direct $v_{\text {inf }}=0.0 \mathrm{~km} / \mathrm{s} \mathrm{lsp}=3000 \mathrm{~s}$

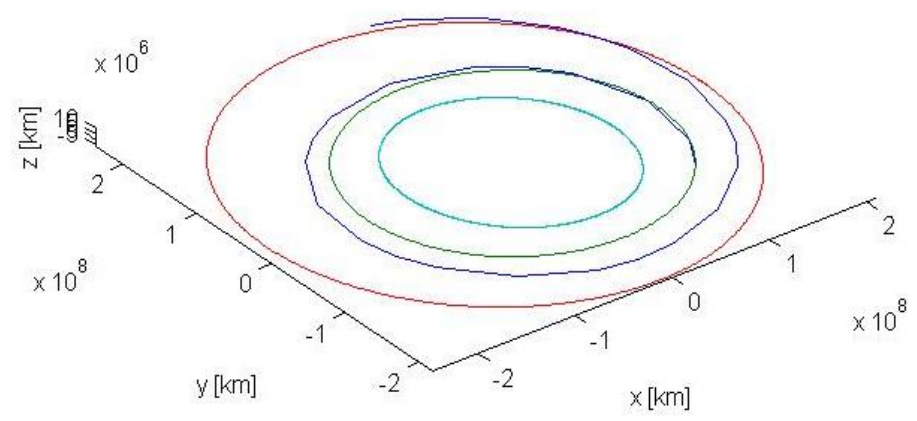

Figure 14: Direct Transfer Trajectory with 220mN maximum thrust

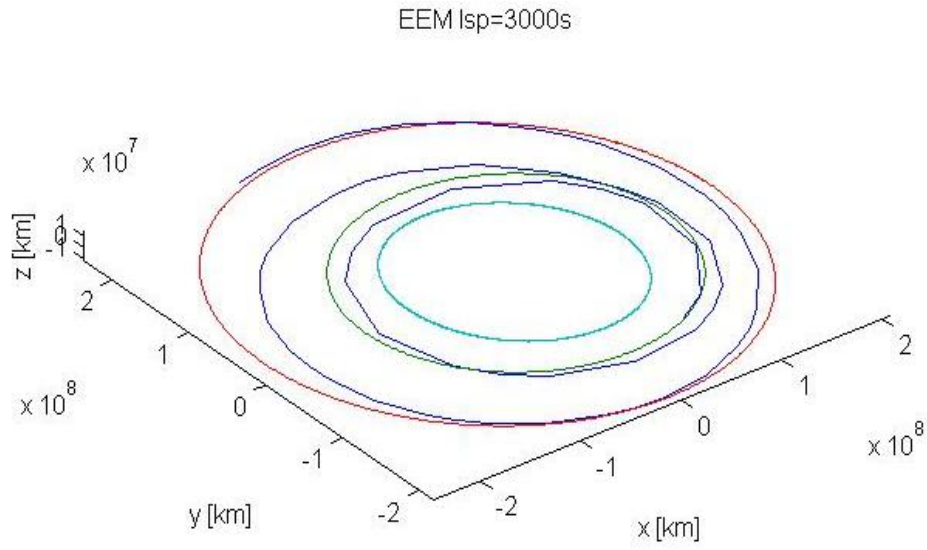

Figure 15: EEM Trajectory with 220mN maximum thrust 

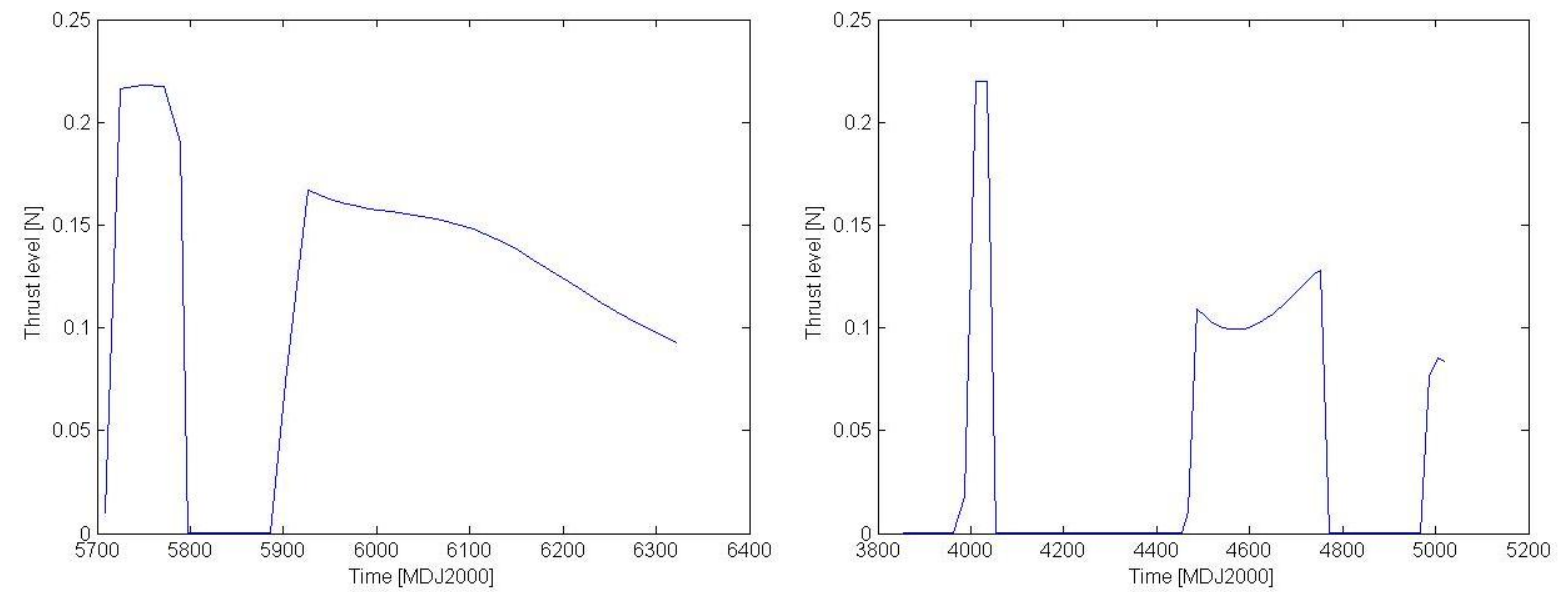

Figure 16: Thrust profile for the direct transfer (left) and the EEM transfer (right) with $220 \mathrm{mN}$ maximum thrust

\subsection{Contingency Analysis}

In order to explore possible recovery options, should the spacecraft suffer motor failure during the course of a mission to enable continuous communications between Earth and Mars, various contingency scenarios were also analyzed. This was combined with a general consideration of how best to utilise the spacecraft between occultations - since the spacecraft are only required to provide a relay service during occultation, maintaining the AEP for a full synodic period is not necessary, and thus one potential strategy is to let the spacecraft drift away from the AEP in between two occultation periods, to conserve fuel. If no contingency occurs, maneuvers can be planned to re-acquire the AEP at minimum propellant cost after one synodic period. For this analysis the maximum thrust level of the spacecraft is assumed to be $80 \mathrm{mN}$ and the Isp is 3000 s. Figure 17 shows the case in which no contingency occurs and maneuvers are planned to leave the leading, or trailing, point and reach the trailing, or leading point. In this case, the two transfers are symmetric.

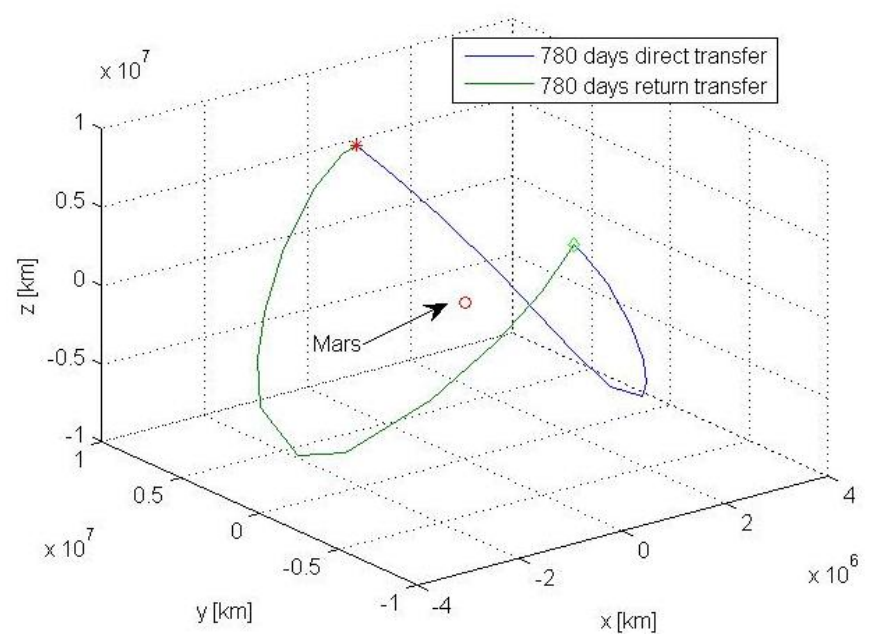

Figure 17: Complete recovery transfer with no drift 
Figure 18 shows the case in which the spacecraft is at the leading AEP (red circle) and experiences a failure for 340 days. The spacecraft drifts to the red star (i.e. along the purple line) where the engines go back online and a recovery is performed to reach the trailing AEP (green line). The same figure shows also the case in which the contingency occurs at the trailing AEP. The spacecraft drifts for 340 days (red line), and then a recovery is performed to reach the leading AEP (light blue line).

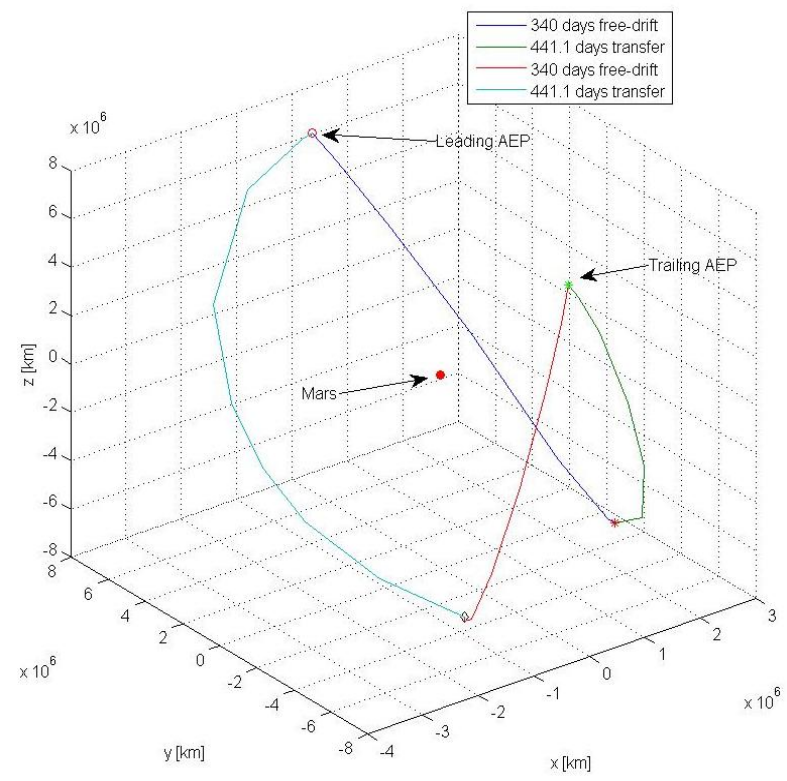

Figure 18: Complete recovery transfer from a 340 days drift

Table 4 provides a numerical summary of these the two cases. In the table the forward transfer refers to the leadingto-trailing transfer and the return transfer refers to the trailing-to-leading transfer.

Table 4: Summary of leading-to-trailing/trailing-to-leading transfer

\begin{tabular}{|l|c|c|c|c|c|}
\hline & $\begin{array}{c}\text { Specific } \\
\text { impulse } \\
\text { Isp }(s)\end{array}$ & $\begin{array}{c}\text { Total propellant } \\
\text { consumed } \\
\mathbf{m}_{\mathbf{p}}(\mathrm{kg})\end{array}$ & $\begin{array}{c}\mathbf{m}_{\mathrm{p}}(\mathbf{k g}) \\
\text { forward } \\
\text { transfer }\end{array}$ & $\begin{array}{c}\mathbf{m}_{\mathrm{p}}(\mathrm{kg}) \\
\text { return } \\
\text { transfer }\end{array}$ & $\begin{array}{c}\text { Time of Flight } \\
(\text { days })\end{array}$ \\
\hline 340 drift case & 3000 & 32.74 & 4.64 & 28.1 & $780+780$ \\
\hline 0 drift case & 3000 & 14.528 & 4.63 & 9.8977 & $780+780$ \\
\hline
\end{tabular}

In the case of a contingency with a recovery after 340 days the leading-to-trailing transfer is quite inexpensive. However, the trailing-to-leading transfer has a substantial cost. The total cost for a roundtrip would be about 32.74 $\mathrm{kg}$ every 1562.2 days. Note that since, in these calculations, the mass of the spacecraft is assumed to be $1000 \mathrm{~kg}$ at the beginning of every transfer, then the total propellant consumption is a slight overestimation of the actual expected cost. 
Table 4 also shows that, if the maneuvers are planned and no contingency occurs (i.e. 0 drift case), the total cost of a roundtrip reduces to about $14.5 \mathrm{~kg}$ every 1562.2 days, or, equivalently, $14.5 \mathrm{~kg}$ in total across two spacecraft switching position in 781.1 days. Therefore, an exchange of position between trailing and leading points is relatively inexpensive.

An alternative scenario is that the spacecraft is maneuver to return to the original AEP. Thus, if no contingency occurs, leading-to-leading transfers and trailing-to-trailing transfers are planned to re-acquire respectively the leading and the trailing AEP. If a contingency occurs, the spacecraft drifts away and after a number of days the recovery maneuver starts. The cost of a recovery maneuver is evaluated for a drift time of 0, 100, 200, 300 and 390 days. The propellant cost is represented in Figure 19, where the drift time is called time-to-intervention, and the resultant analysis shows that if no contingency occurs, an AEP-to-AEP transfer has a minimal cost of less than $8 \mathrm{~kg}$, for the roundtrip, for the leading point and less than $5 \mathrm{~kg}$ roundtrip for the trailing point. In the case of a failure at the leading point, the cost can grow up to $50 \mathrm{~kg}$ roundtrip while it remains contained for a failure at the trailing point. In the former case the spacecraft flies around Mars before re-acquiring the AEP, in the case of a drift time of 390 days. Therefore, if a single spacecraft is used and a contingency occurs at the leading point a leading-to-trailing transfer is recommended. Vice versa, if a contingency occurs at the trailing point a trailing-to-trailing transfer is recommended.

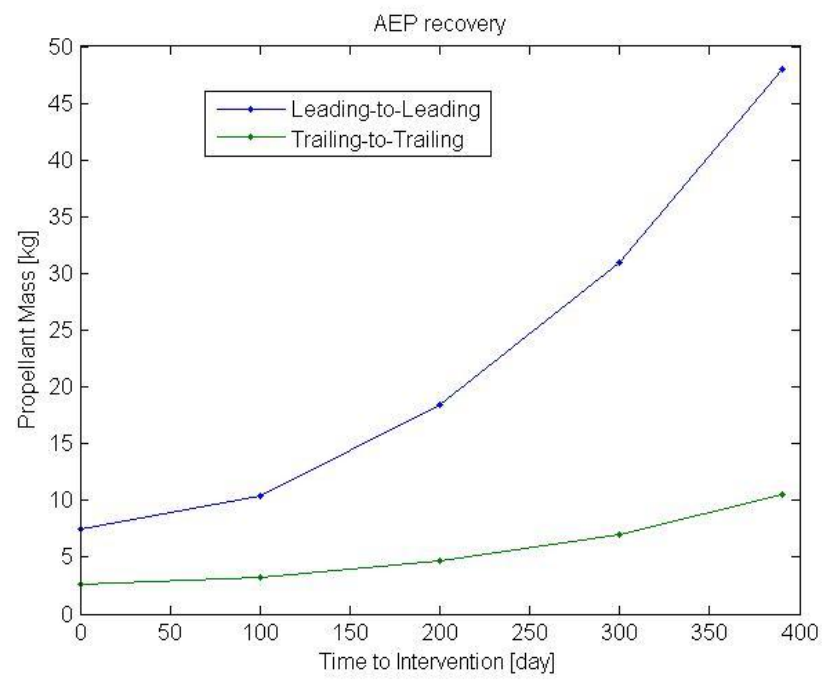

Figure 19: Propellant cost against time-to-intervention for AEP-AEP contingency transfer

This analysis optimizes the return to the AEP to minimize the propellant consumption. However, the return time (time to go from one AEP back to the same AEP), in some cases, could be 681 days, i.e. 100 days before the next occultation. This situation is unfavorable because the spacecraft would need to maintain the AEP with constant thrust for an extra 100 days every synodic period, in addition to the 90 day-burn already planned for during the 
occultation. An approximate estimate suggests thrusting at the AEP for the extra 100 days would require around $23 \mathrm{~kg}$ of propellant, in addition to the less than 8 or $5 \mathrm{~kg}$ required to do this fuel-efficient transfer, resulting in total propellant consumptions of $31 \mathrm{~kg}$ and $28 \mathrm{~kg}$ (approximately).

Instead, the spacecraft can be forced to return to the AEP after 1 synodic period exactly. Figure 20 shows the transfer trajectories for different drift times, while Figure 21 shows the propellant consumption for different drift times. From Figure 21, we can see that maintaining the AEP is not convenient as the whole return maneuver requires a shorter total thrust time. It is also interesting to note that for the trailing AEP the cost remains almost constant (the variation is between 24 and $25 \mathrm{~kg}$ ) for different drift times.

a)
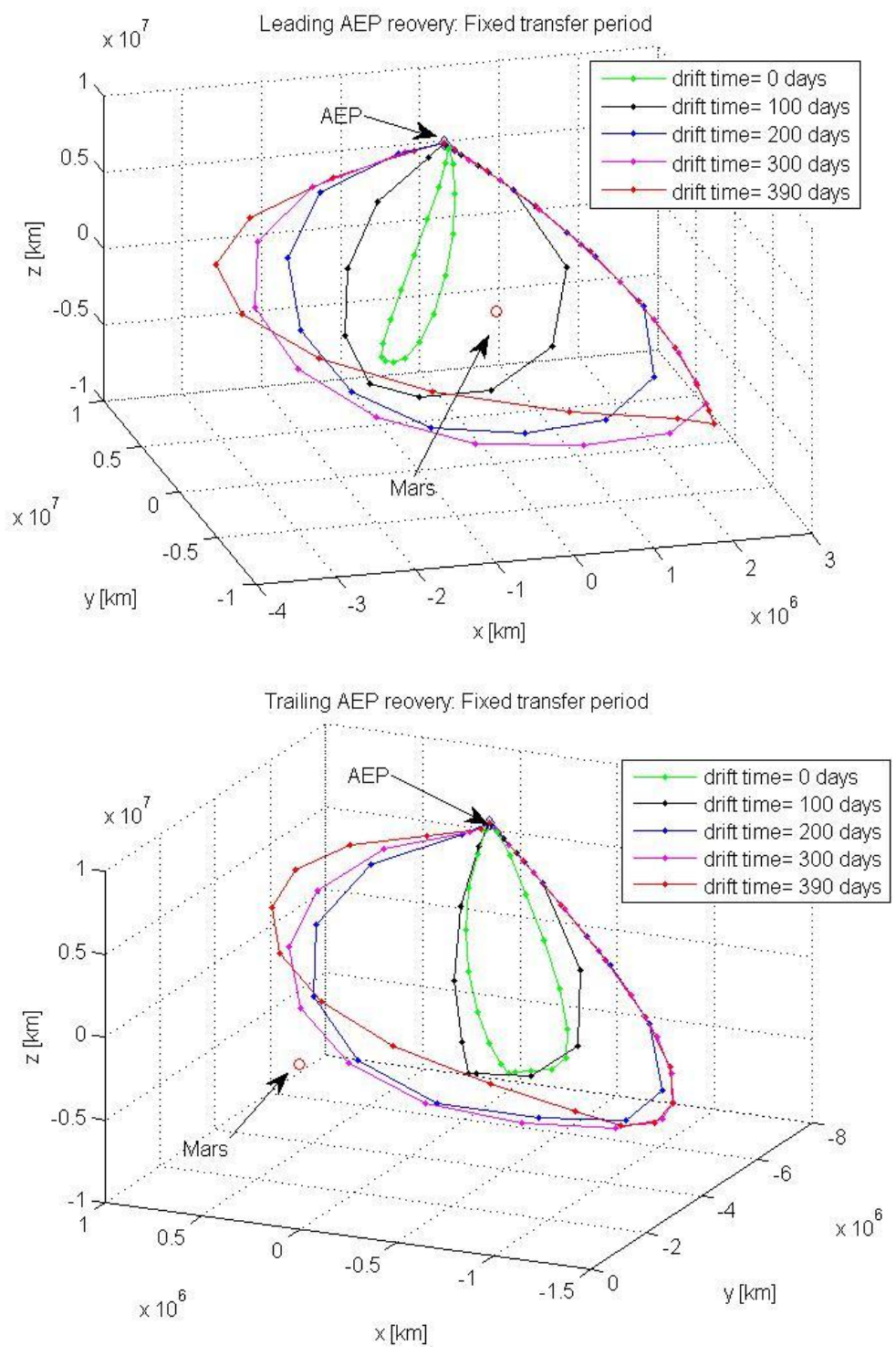

Figure 20: Families of AEP-AEP contingency transfers with fixed return period: a) leading-to-leading, b) trailing-to-trailing. 


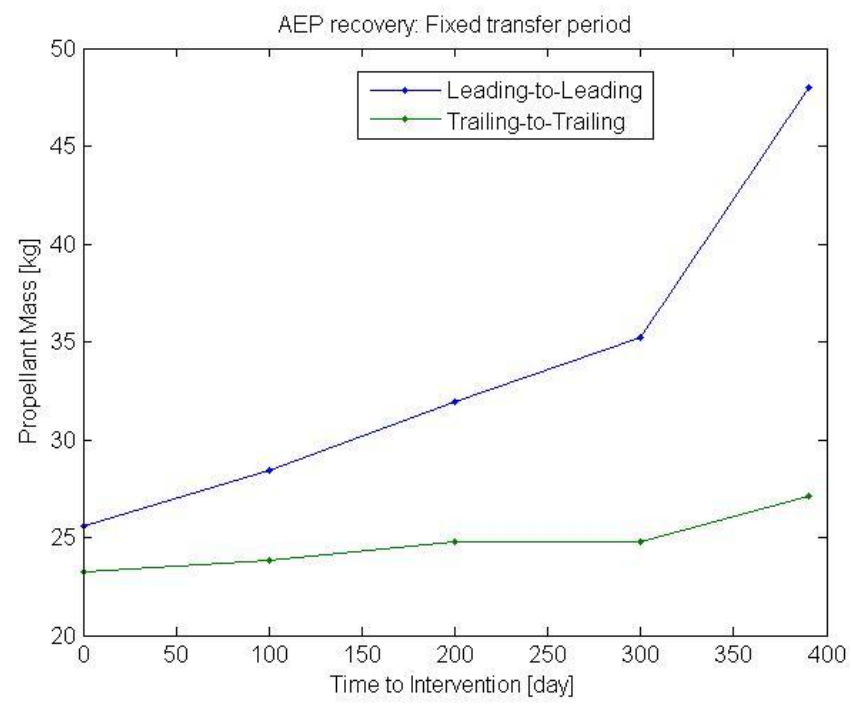

Figure 21: Propellant cost against time-to-intervention for AEP-AEP contingency transfer. Fixed return period.

Fixing the leading-to-leading or trailing-to-trailing re-acquire time to 1 synodic period results in propellant consumptions of 25 and $24 \mathrm{~kg}$, approximately, which is clearly more efficient overall than the previous situation where the transfer trajectory itself is optimized for fuel efficiency but the spacecraft comes back to the AEP too early and has to expend a lot of energy to stay there. However it must be noted that this is still less efficient than the AEP-swapping system, which requires a total of around $14.5 \mathrm{~kg}$ for two spacecraft to switch position from leadingto-trailing and vice-versa in one synodic period. Again, if the engine fails to thrust, the situation can be recovered with increasing amounts of propellant for ever-increasing failure times, although interestingly only for the leadingto-leading case (where the increase is dramatic, as per before), with it being approximately constant for the trailingto-trailing case.

Note that, all the trajectories calculations in this section were performed with a relatively coarse grid of nodes for the finite elements. More accurate numbers can be obtained with a finer grid (the work already being in progress) although the general conclusions would not change.

\section{$\underline{5-\text { Summary and Conclusions }}$}

A potential Earth-Mars communication relay has been outlined, designed to ensure continuous communications between the two planets during periods of solar occultation. The relay makes use of highly non-Keplerian orbits, using a continuous low thrust propulsion of approximately the same order as that of the gravitational acceleration experienced to maintain a "hover" point above Mars. 
Both pure-SEP and hybrid SEP/sail spacecraft were considered for the relay. In the latter case, the addition of a reasonable near-term solar sail only proves advantageous for the case of a communications relay using Ka-band, and particularly only for communication with assets stationed near the poles, during summertime. For a communication relay using X-band and a polesitter position, hybrid propulsion would require a significantly advanced solar sail if a reasonable level of SEP thrust is assumed. This is partly due to the poor performance of solar sails high out of the orbital plane, and partly due to the poor performance of solar sails far away from the Sun. For these reasons it is suggested that hybrid propulsion would make better use of highly non-Keplerian orbits within the orbital plane and within the vicinity of celestial bodies closer to the Sun, with the GeoStorm mission being one example briefly shown to be potentially enhanced by hybrid propulsion.

Considering various different insertion options for a pure-SEP mission, it seems quite feasible that low-thrust propulsion can be used to insert a spacecraft $0.176 \mathrm{AU}$ directly above Mars, although providing the power for such a spacecraft would be an engineering challenge. However reducing the thrust requirements, by requiring the relay spacecraft to be displaced less far out of the orbital plane - by both the addition of a second spacecraft to the relay and utilising Ka-band radio communication - can significantly ease this problem. Further, the Astrium RIT-XT engine seems more ideal than the Qinetiq T6-based one considered initially, operating as it does at 3000s with a $22 \mathrm{~W} / \mathrm{mN}$ of specific power, and thus giving the required area of the solar arrays to $39.1 \mathrm{~m}^{2}$ (with a $500 \mathrm{~W}$ total subsystem power requirement and a $20 \%$ margin). If this system can provide $220 \mathrm{mN}$ thrust at $1 \mathrm{AU}$, it would correspond to $80 \mathrm{mN}$ at the aphelion of Mars - exactly what is needed for the lower-thrust Ka-band relay architecture option with the relay spacecraft displaced 45 degrees out of the orbital plane. Getting there then with this system seems to be a simple trade-off, with a direct variable thrust option using almost twice the propellant but taking half as long in flight time compared to an EEM transfer. Both options however were tuned to provide $1000 \mathrm{~kg}$ wet mass at Mars.

Various contingency schemes were analyzed for once the spacecraft is in position, with it being shown that transferring the spacecraft from a leading orbit to a trailing orbit is relatively inexpensive (around $4.6 \mathrm{~kg}$ of propellant), although the opposite transfer being considerably more so, requiring nearer $10 \mathrm{~kg}$ of propellant. If there is a motor failure, however, although the situation can be recovered if the engines can be fired again, the amount of propellant required increases dramatically to over $32 \mathrm{~kg}$ of propellant for the roundtrip, assuming the engine is offline for 340 days. These transfers take one synodic period to complete, which does not account for the time actually spent at the AEP, but provides a useful insight of the propellant consumption required regardless.

Alternatively the spacecraft can re-acquire the same AEP, i.e. leading-to-leading or trailing-to-trailing. The most fuel efficient way to carry out this transfer would require less than $8 \mathrm{~kg}$ for the leading-to-leading case and less than $5 \mathrm{~kg}$ for the trailing-to-trailing case. However these transfers are not fixed period in length, although they are shorter than one synodic period, and would thus potentially require the spacecraft to be thrusting at the AEP for a few days before the next occultation, and hence using a small amount of extra fuel that would have to be accounted for in a 
more detailed study. Performing the same analysis and fixing the leading-to-leading or trailing-to-trailing re-acquiry time to 1 synodic period, as direct comparisons to both the free period transfer case and the AEP-swapping case of the same period, results in propellant consumptions of 25 and $24 \mathrm{~kg}$, approximately, which is clearly less efficient than the AEP-swapping system (which requires a total of around $14.5 \mathrm{~kg}$ for two spacecraft to switch position from leading-to-trailing and vice-versa in one synodic period), although again it must be stressed these values do not take into account the on-station time at the AEP required to maintain the communications relay during occultations. Again, if the engine fails to thrust, the situation can be recovered with increasing amounts of propellant for everincreasing failure times, although interestingly only for the leading-to-leading case (where the increase is dramatic, as per before), with it being approximately constant for the trailing-to-trailing case.

\section{Acknowledgments}

This work was part-funded by ESA Contract Number 22349/09/F/MOS; Study on Gravity Gradient Compensation Using Low Thrust High Isp Motors.

\section{References}

[1] Dusek, H. M., "Motion in the Vicinity of Libration Points of a Generalized Restricted Three-Body Model", Methods in Astrodynamics and Celestial Mechanics: Progress in Astronautics and Aeronautics, Vol. 17, pp. 37-54, 1966

[2] McInnes, C. R., McDonald, A. J. C., Simmons, J. F. L., and MacDonald, E. W., "Solar Sail Parking in Restricted Three-Body Systems", Journal of Guidance, Dynamics and Control, Vol. 17, No. 2, pp. 399-406, 1994

[3] Forward, R. L., "Statite: A Spacecraft That Does Not Orbit”, Journal of Spacecrafts and Rockets, Vol. 28, No. 5, pp. 606-611, 1991

[4] McInnes, C. R., Solar Sailing: Technology, Dynamics and Mission Applications, Praxis Publishing, Chichester, 1999, ISBN $1-85233-102-\mathrm{X}$

[5] West, J. L., "The GeoStorm Warning Mission: Enhanced Opportunities Based On New Technology", 14 $4^{\text {th }}$ AAS/AIAA Spaceflight Mechanics Conference, Paper AAS 04-102, Maui, Hawaii, Feb $8^{\text {th }}-12^{\text {th }}, 2004$

[6] West, J. L., "Solar Sail Vehicle System Design for the GeoStorm Warning Mission", Structures, Structural Dynamics and Materials Conference, Atlanta, USA, April 2000

[7] Morimoto, M. Y., Yamakawa, H., and Uesugi, K., "Artificial Equilibrium Points in the Low-Thrust Restricted Three-Body Problem", Journal of Guidance, Control, and Dynamics, Vol. 30, No. 5, pp. 1563-1567, 2007

[8] Morimoto, M. Y., Yamakawa, H., and Uesugi, K, "Periodic Orbits with Low-Thrust Propulsion in the Restricted Three-Body Problem", Journal of Guidance, Control, and Dynamics, Vol. 29, No. 5, pp. 1131-1139, 2006

[9] Spilker, T. R., “Saturn Ring Observer”, Acta Astronautica, Vol. 52, pp. 259-265, 2003

[10] McKay, R. J., Macdonald, M., Bosquillon de Frescheville, F., Vasile, M., McInnes, C. R., and Biggs, J. D., "Non-Keplerian Orbits Using Low Thrust, High ISP Propulsion Systems", In 60th International Astronautical Congress, IAC Paper 09.C1.2.8, Daejeon, Republic of Korea, 12 - 16 October, 2009 
[11] Wawrzyniak, G. G., and Howell, K. C., "The Solar Sail Lunar Relay Station: An Application of Solar Sails in the EarthMoon System", In 59th International Astronautical Congress, IAC Paper 08.C1.3.14, Glasgow, Scotland, 29 September - 03 October 2008

[12] McInnes, C. R., "Dynamics, Stability and Control of Displaced Non-Keplerian Orbits", Journal of Guidance, Control, and Dynamics, Vol. 21, No. 5, September-October 1998

[13] McKay, R. J., and Macdonald, M., "Study on Gravity Gradient Compensation Using Low Thrust High Isp Motors", Technical Notes GGC.UofS.TN.0001 and GGC.UofS.TN.0001a, ESA Contract No 22349/09/F/MOS, February 2010

[14] Wallace, N., "Testing of the Qinetiq T6 Thruster in Support of the ESA BepiColombo Mercury Mission", Proceedings of the $4^{\text {th }}$ International Spacecraft Propulsion Conference (ESA SP-555), Chia Laguna, Cagliari, Italy, $2^{\text {nd }}-9^{\text {th }}$ June 2004

[15] Shambayati, S., Morabito, D., Border, J. S., Davarian, F., Lee, D., Mendoza, R., Britcliffe, M., and Weinreb, S., "Mars Reconnaissance Orbiter Ka-Band (32 GHz) Demonstration: Cruise Phase Operations", The Proceedings of the AIAA SpaceOps 2006 Conference, Rome, Italy, June 19-24, 2006

[16] Macdonald, M., and McInnes, C. R., "Solar Sail Mission Applications and Future Advancement", $2^{\text {nd }}$ International Symposium on Solar Sailing, ISSS 2010, New York, USA, July 20-22, 2010

[17] Leipold, M., and, Götz, M., "Hybrid Photonic/Electric Propulsion", Kayser-Threde, TR SOL4-TR-KTH-0001, Munich, ESA Contract No. 15334/01/NL/PA, January 2002

[18] Mengali, G., and Quarta, A. A., "Trajectory Design with Hybrid Low-Thrust Propulsion System", Journal of Guidance, Control, and Dynamics, Vol. 30, No. 2, pp. 419-426, 2007

[19] Baig, S., and McInnes, C. R., "Artificial Three-Body Equilibria for Hybrid Low-Thrust Propulsion”, Journal of Guidance, Control, and Dynamics, Vol. 31, No. 6, pp. 1644-1655, 2008

[20] Simo, J., and McInnes, C. R., "Designing Displaced Lunar Orbits Using Low Thrust Propulsion", Journal of Guidance, Control, and Dynamics, Vol. 33, No. 1, pp. 259-265, 2010

[21] Ceriotti, M., and McInnes, C. R., "A Near Term Pole-Sitter Using Hybrid Solar Sail Propulsion", $2^{\text {nd }}$ International Symposium on Solar Sailing, ISSS 2010, New York, USA, July 20-22, 2010

[22] McInnes, C. R., "Artificial Lagrange Points for a Partially Reflecting Flat Solar Sail”, Journal of Guidance, Control and Dynamics, Vol. 22, No. 1, pp. 185-187, 1999

[23] Dachwald, B., Macdonald, M., McInnes, C. R., Mengali, G., and Quarta, A. A., "Impact of Optical Degradation on Solar Sail Mission Performance", Journal of Spacecraft and Rockets, Vol. 44, No. 4, pp. 740-749, 2007

[24] Leipold, M., Seboldt, W., Lingner, S., Borg, E., Herrmann, A., Pabsch, A., Wagner, O., and Brückner, J., "Mercury Sunsynchronous Polar Orbiter with a Solar Sail”, Acta Astronautica, Vol. 39, Issues 1-4, pp. 143-151, 1996

[25] Vasile M., Bernelli-Zazzera F., Fornasari N., Masarati P., "Design of Interplanetary and Lunar Missions Combining LowThrust and Gravity Assists", Final Report of the ESA/ESOC Study Contract No. 14126/00/D/CS, September 2002

[26] Vasile M and Bernelli-Zazzera F, "Optimizing Low-Thrust and Gravity Assist Manoeuvres to Design Interplanetary Trajectories", Journal of the Astronautical Sciences, Vol. 51, No. 1, 2003

[27] Vasile M, Bottasso C L, and Finzi A E, "Lunar Orbital Dynamics by Finite Element in Time Method", Aerotecnica Missilie Spazio, Vol. 75, No. 3/4, 1996

[28] D'Accolti, G., Beltrame, G., Ferrando, E., Brambilla, L., Contini, R., Vallini, L., Mugnuolo, R., Signorini, C., Caon, A., and Fiebrich, H., "The Solar Array Photovoltaic Assembly for the Rosetta Orbiter and Lander Spacecrafts", Space Power, Proceedings of the Sixth European Conference (p.445, ESA SP-502), Porto, Portugal, $6^{\text {th }}-10^{\text {th }}$ May 2002

[29] Racca, G. D., Marini, A., Stagnaro, L., van Dooren, J., di Napoli, L., Foing, B. H., Lumb, R., Volp, J., Brinkmann, J., Grünagel, R., Estublier, D., Tremolizzo, E., McKay, M., Camino, O., Schoemaekers, J., Hechler, M., Khan, M., Rathsman, P., 
Andersson, G., Anflo, K., Berge, S., Bodin, P., Edfors, A., Hussain, A., Kugelberg, J., Larsson, N., Ljung, B., Meijer, L., Mörtsell, A., Nordebäck, T., Persson, S., and Sjöberg, F., "SMART-1 Mission Description and Development Status", Planetary and Space Science, Vol. 50, Issues 14-15, pp. 1323-1337, 2002. 